\title{
LOCAL LANGLANDS AND SPRINGER CORRESPONDENCES
}

\author{
ANNE-MARIE AUBERT
}

\begin{abstract}
This notes are the written version of a course given by the author at the workshop "Representation theory of $p$-adic groups" that was held at IISER Pune, India, in July 2017 (to appear in Representations of p-adic groups: Contributions from Pune, Progress in Math., Birkhäuser).

They give notably an overview of results obtained jointly with Ahmed Moussaoui and Maarten Solleveld on the local Langlands correspondence, focusing on the links of the latter with both the generalized Springer correspondence and the geometric conjecture, the so-called ABPS Conjecture, introduced in collaboration with Paul Baum, Roger Plymen and Maarten Solleveld.
\end{abstract}

\section{INTRODUCTION}

The local Langlands correspondence predicts a relation between two rather different kinds of objects: on one side irreducible representations of reductive groups over a local field $F$, on the other side certain analogs of Galois representations, called Langlands parameters.

Let $G$ be the group of $F$-rational points $G$ of a connected reductive algebraic group $\mathbf{G}$ over a non Archimedean local field $F$. Slightly more precisely, the local Langlands conjecture for $G$ asserts the existence a finite to one surjection

$$
\operatorname{rec}_{G}: \operatorname{Irr}(G) \rightarrow \Phi(G),
$$

from the set $\operatorname{Irr}(G)$ of (isomorphism classes of) irreducible smooth representations of $G$ to the set $\Phi(G)$ of equivalence classes (for a certain equivalence relation) of Langlands parameters for $G$, that satisfies a certain list of chosen, expected properties.

The (conjectural) fibers of $\operatorname{rec}_{G}$ are called $L$-packets for $G$. The irreducible representations $\pi$ inside a given $L$-packet are said to be $L$-indistinguishable. To distinguish them, the idea (see Lusztig [Lus1], Vogan [Vog, Arthur [Ar1]) is to "enhance" $\phi$ by an additional datum $\rho$, which is an irreducible representation of a certain finite group $\mathcal{S}_{\phi}$ (defined in Equation (3) below). The pair $(\phi, \rho)$ will be called an enhanced Langlands parameter for $G$. Let $\Phi_{\mathrm{e}}\left(G^{\sharp}\right)$ be the collection of equivalence classes of such pairs $(\phi, \rho)$ with $\rho \in \operatorname{Irr}\left(\mathcal{S}_{\phi}\right)$. Then the LLC for $G$ should be an injective map

$$
\operatorname{Irr}(G) \rightarrow \Phi_{\mathrm{e}}(G),
$$

which satisfies several natural properties. The map will almost never be surjective, but for every $\phi$ which is relevant for $G$ the image should contain at least one pair $(\phi, \rho)$.

2010 Mathematics Subject Classification. 20C08,14F43,20G20.

It is a pleasure to acknowledge the excellent comments and questions of the participants of the workshop "Representation theory of $p$-adic groups" at IISER Pune. 
A remarkable aspect of the Langlands conjecture $\mathrm{Vog}$ is that it is better to consider not just one reductive group $G$ at a time, but all inner forms (more precisely all inner twists) $G_{\vartheta}$ of $G$ simultaneously. Inner twists share the same Langlands dual group. The hope is that one can turn (1) into a bijection by defining a suitable equivalence relation on the set of inner twists and taking the corresponding union of the sets $\operatorname{Irr}\left(G_{\vartheta}\right)$ on the left hand side. Such a statement was proven for unipotent representations (also known as representations with unipotent reduction) of simple $p$-adic groups in Lus5.

The main goal of these lectures is to provide an overview of the main results obtained jointly with Ahmed Moussaoui and Maarten Solleveld in AMS1 on the local Langlands correspondence, focusing on the links of the latter with both the generalized Springer correspondence and the geometric conjecture, the so-called ABPS Conjecture, introduced in collaboration with Paul Baum, Roger Plymen and Maarten Solleveld and studied in several articles, notably ABPS7. We give also an account of the known results regarding the preservation (and non-preservation) of the depth by the LLC.

For maximal generality, we adhere to the setup for $L$-parameters used by Arthur in [Ar1]. Let $W_{F}$ be the Weil group of $F$, let ${ }^{L} G=G^{\vee} \rtimes W_{F}$ be the $L$-group of $G$. Let $G_{\mathrm{ad}}^{\vee}$ be the adjoint group of $G^{\vee}$, and let $G_{\mathrm{sc}}^{\vee}$ be the simply connected cover of the derived group of $G_{\mathrm{ad}}^{\vee}$. Let $\phi: W_{F} \times \mathrm{SL}_{2}(\mathbb{C}) \rightarrow{ }^{L} G$ be an $L$-parameter, let $\mathrm{Z}_{G_{\mathrm{ad}}}^{\vee}\left(\phi\left(W_{F}\right)\right)$ be the centralizer of $\phi\left(W_{F}\right)$ in $G_{\mathrm{ad}}^{\vee}$ and let

$$
\mathcal{G}_{\phi}:=\mathrm{Z}_{G_{\mathrm{sc}}^{\vee}}^{1}\left(\phi\left(W_{F}\right)\right)
$$

be its inverse image in $G_{\mathrm{sc}}^{\vee}$. To $\phi$ we associate the finite group

$$
\mathcal{S}_{\phi}:=\pi_{0}\left(\mathrm{Z}_{G_{\mathrm{sc}}^{\vee}}^{1}(\phi)\right)
$$

where $\mathrm{Z}^{1}$ is again defined via $G_{\mathrm{ad}}^{\vee}$. We call any irreducible representation of $\mathcal{S}_{\phi}$ an enhancement of $\phi$. The group $\mathcal{S}_{\phi}$ coincides with the group considered by both Arthur in [Ar1] and Kaletha in [Kal1, §4.6]. A remarkable fact is that the group $\mathcal{S}_{\phi}$ is isomorphic to the component group

$$
A_{\mathcal{G}_{\phi}}\left(u_{\phi}\right):=\pi_{0}\left(\mathrm{Z}_{\mathcal{G}_{\phi}}\left(u_{\phi}\right)\right)
$$

where $u_{\phi}:=\phi\left(1,\left(\begin{array}{ll}1 & 1 \\ 0 & 1\end{array}\right)\right)$. It provides a way to plug the generalized Springer correspondences for the groups $\mathcal{G}_{\phi}$, where $\phi$ runs over $\Phi(G)$, in the study of the local Langlands correspondence for $G$.

In particular, it allows to transfer the Lusztig notion of cuspidality for representations of the groups $A_{\mathcal{G}_{\phi}}\left(u_{\phi}\right)$ into a notion of cuspidality for enhanced $L$-parameters for $G$ : an enhanced $L$-parameter $(\phi, \rho)$ for $G$ is called cuspidal if $u_{\phi}$ and $\rho$, considered as data for the complex reductive group $\mathcal{G}_{\phi}$, form a cuspidal pair. By definition this means that the restriction of $\rho$ from $A_{\mathcal{G}_{\phi}}\left(u_{\phi}\right)$ to $A_{\mathcal{G}_{\phi}^{\circ}}\left(u_{\phi}\right)$ is a direct sum of cuspidal representations in Lusztig's sense [Lus2]. Intuitively, it says that $\rho$ or $\left.\rho\right|_{A_{\mathcal{G}_{\phi}^{\circ}}\left(u_{\phi}\right)}$ cannot be obtained (via an appropriate notion of parabolic induction) from any pair $\left(u^{\prime}, \rho^{\prime}\right)$ that can arise from a proper Levi subgroup of $\mathcal{G}_{\phi}^{\circ}$. It is essential to use $L$-parameters enhanced with a representation of a suitable component group, for cuspidality cannot be detected from the $L$-parameter alone.

We conjecture in [AMS1, $\S 6$ ] (generalizing to $G$ arbitrary the conjecture stated by Moussaoui when $G$ is $F$-split: [Mou1, Conjecture 1.2]) that the cuspidal enhanced 
Langlands parameters correspond by the LLC to the irreducible supercuspidal representations of $G$.

The validity of this conjecture is proved for representations with unipotent reduction of the group $G$ of the $F$-rational points of any connected reductive algebraic group which splits over an unramified extension of $F$ in [FOS, Theorem 2] (when $G$ is simple of adjoint type it is a special case of [Lus4, [Lus5]), for the DeligneLusztig depth-zero supercuspidal representations (as a consequence of [DeRe]), and also for general linear groups and split classical $p$-adic groups (any representation) (see [Mou1]), for inner forms of linear groups and of special linear groups, and for quasi-split unitary $p$-adic groups (any representation) (see [AMS1, §6]).

\section{Contents}

1. Introduction 1

2. Langlands parameters 3

2.1. The Weil group 3

2.2. The $L$-group 5

\begin{tabular}{lll}
\hline 2.3. & Definitions for Langlands parameters & 7
\end{tabular}

2.4. L-packets 8

\begin{tabular}{lll}
\hline 3. & Enhanced Langlands parameters & 9
\end{tabular}

$\begin{array}{llr}3.1 . & \text { The group } \mathcal{S}_{\phi} & 9\end{array}$

$\begin{array}{lll}3.2 . & \text { Definition of enhanced Langlands parameters } & 10\end{array}$

\begin{tabular}{lll}
\hline 3.3. & Desiderata for the local Langlands correspondence & 11
\end{tabular}

$\begin{array}{lll}3.4 . \quad \text { Depth } & 13\end{array}$

\begin{tabular}{lrr}
\hline $4 . \quad$ Generalized Springer correspondence & 16
\end{tabular}

\begin{tabular}{lll}
\hline 4.1. & Cuspidal enhanced unipotent classes & 16
\end{tabular}

4.2. A partition of the enhancement of the unipotent variety of $\mathcal{G}^{\circ} \quad 18$

\begin{tabular}{lll}
\hline 4.3. & Twisted group algebras & 19
\end{tabular}

\begin{tabular}{lll}
\hline 4.4. & A partition of the enhancement of the unipotent variety of $\mathcal{G}$ & 19
\end{tabular}

5. Cuspidality for enhanced $L$-parameters: definition and conjecture 20

6. A partition of the set of enhanced Langlands parameters 20

6.1. A generalized Springer correspondence for enhanced $L$-parameters 22

$\begin{array}{lll}\text { 7. A Galois version of the ABPS Conjecture } & 22\end{array}$

\begin{tabular}{lll}
\hline 7.1. & Twisted extended quotients & 22
\end{tabular}

7.2. The Bernstein decomposition of the category of smooth representations 23

$\begin{array}{lll}7.3 . & \text { The ABPS Conjecture } 24\end{array}$

$\begin{array}{lll}7.4 . & \text { A version for enhanced } L \text {-parameters } & 25\end{array}$

\begin{tabular}{lll}
\hline $7.5 . \quad$ A conjectural diagram & 26
\end{tabular}

\begin{tabular}{ll}
\hline References & 27
\end{tabular}

\section{LANGLANDS PARAMETERS}

2.1. The Weil group. Let $F$ be a local non-Archimedean field with finite residual field $k_{F}=\mathbb{F}_{q}$. Let $F_{\text {sep }}$ be a fixed separable closure of $F$, and let $\Gamma_{F}$ denote the Galois group of $F_{\text {sep }} / F$. The field $F$ admits a unique unramified extension $F_{m} / F$ of degree $m$ and contained in $F_{\text {sep }}$, for each integer $m \geq 1$. The composite of all the fields $F_{m}$ is the unique maximal unramified extension of $F$ contained in $F_{\text {sep }}$ and 
will be denoted by $F_{\text {ur }}$. The extension $F_{\text {ur }}$ will allow us to decompose the study of $\Gamma_{F}$ in two steps of different nature by considering separately the group $\operatorname{Gal}\left(F_{\mathrm{ur}} / F\right)$ and the group $I_{F}:=\operatorname{Gal}\left(F_{\mathrm{sep}} / F_{\mathrm{ur}}\right)$, that is called the inertia group of $F$ : we have an exact sequence of topological groups

$$
1 \rightarrow I_{F} \rightarrow \Gamma_{F} \rightarrow \operatorname{Gal}\left(F_{\mathrm{ur}} / F\right) \rightarrow 0 .
$$

The extension $F_{m} / F$ is Galois and the group $\operatorname{Gal}\left(F_{m} / F\right)$ is cyclic (that is, generated by a single element). An $F$-automorphism of $F_{m}$ is determined by its action on the residual field $k_{F_{m}} \simeq \mathbb{F}_{q^{m}}$ of $F_{m}$, and there is a unique element $\sigma_{m}$ of $\operatorname{Gal}\left(F_{m} / F\right)$ which acts on $k_{F_{m}}$ by the elevation at the power $q$. We set $\mathrm{Fr}_{m}:=\sigma_{m}^{-1}$. The map $\mathrm{Fr}_{m} \mapsto 1+m \mathbb{Z}$ gives a canonical isomorphism from $\operatorname{Gal}\left(F_{m} / F\right)$ onto $\mathbb{Z} / m \mathbb{Z}$. Taking the inverse limit over $m$, we get a canonical isomorphism of topological groups between $\operatorname{Gal}\left(F_{\text {ur }} / F\right)$ and

$$
\widehat{\mathbb{Z}}:=\lim _{m \geq 1} \mathbb{Z} / m \mathbb{Z}
$$

and a unique element $\operatorname{Fr}_{F} \in \operatorname{Gal}\left(F_{\text {ur }} / F\right)$ which acts on $F_{m}$ as $\operatorname{Fr}_{m}$, for all $m$. The element $\operatorname{Fr}_{F}$ is called the geometric Frobenius substitution on $F_{\text {ur }}$ (its inverse $\sigma_{F}$ is the arithmetic Frobenius substitution). An element of $\Gamma_{F}$ is called a geometric Frobenius element (over $F$ ) if its image in $\operatorname{Gal}\left(F_{\text {ur }} / F\right)$ is $\operatorname{Fr}_{F}$. The Chinese Remainder Theorem gives a canonical isomorphism of topological groups $\widehat{\mathbb{Z}} \simeq \prod_{\ell} \mathbb{Z}_{\ell}$, where $\ell$ range over all prime numbers, and $\mathbb{Z}_{\ell}$ is the (additive) group of $\ell$-adic integers.

We recall some properties of the ramification groups (with respect to the upper numbering) of $\Gamma_{F}$, as defined in [Ser, Remark IV.3.1]:

- $\Gamma_{F}^{-1}:=\Gamma_{F}$ and $\Gamma_{F}^{0}:=I_{F}$, the inertia group.

- For every $r \in \mathbb{R}_{\geq 0}, \Gamma_{F}^{l}$ is the compact subgroup of $I_{F}$ that consists of all $\gamma \in \Gamma_{F}$ which, for every finite Galois extension $E$ of $F$ contained in $F_{\text {sep }}$, act trivially on the ring $\mathfrak{o}_{E} / \mathfrak{p}_{E}^{i(r, E)}$ (where $i(r, E) \in \mathbb{Z}_{\geq 0}$ can be found with Ser, $\S$ IV.3]).

- $r \in \mathbb{R}_{\geq 0}$ is called a jump of the filtration if

$$
\Gamma_{F}^{r+}:=\bigcap_{t>r} \Gamma_{F}^{t}
$$

does not equal $\Gamma_{F}^{r}$. The set of jumps of the filtration is countably infinite and need not consist of integers.

In order to formulate the Langlands correspondence we need to introduce the Weil group $W_{F}$, a subgroup of $\Gamma_{F}$. Let a $W_{F}$ denote the inverse image in $\Gamma_{F}$ of the cyclic subgroup of $\operatorname{Gal}\left(F_{\mathrm{ur}} / F\right)$ generated by $\mathrm{Fr}_{F}$. Then ${ }_{\mathrm{a}} W_{F}$ is the dense subgroup of $\Gamma_{F}$ generated by the geometric Frobenius elements. It is normal in $\Gamma_{F}$ and fits into an exact sequence (of abstract groups)

$$
1 \rightarrow I_{F} \rightarrow{ }_{\mathrm{a}} W_{F} \rightarrow \mathbb{Z} \rightarrow 0 .
$$

The Weil group $W_{F}$ of $F$ (relative to $F_{\text {sep }}$ ) is the topological group, with underlying abstract group a $W_{F}$, so that $I_{F}$ is an open subgroup of $W_{F}$, and the topology on $I_{F}$, as subspace of $W_{F}$, coincides with its natural topology as $\operatorname{Gal}\left(F_{\text {sep }} / F_{\text {ur }}\right) \subset \Gamma_{F}$. Thus $W_{F}$ is locally profinite, and the identity map $\iota_{F}: W_{F} \rightarrow{ }_{\mathrm{a}} W_{F} \subset \Gamma_{F}$ is continuous.

The definition of $W_{F}$ does depend on the choice of $F_{\text {sep }} / F$, but only up to inner automorphism of $\Gamma_{F}$. 
We will need some basic aspects of the representation theory of $W_{F}$. The group $\Gamma_{F}$ being profinite, its smooth representations are semisimple. On the contrary, $W_{F}$ has smooth representations which are not semisimple. The irreducible representations of $W_{F}$ are quite closely related to those of $\Gamma_{F}$ (in particular, they have finite dimension):

(1) if $\tau$ is an irreducible representation of $\Gamma_{F}$, then $\tau \circ \iota_{F}$ is an irreducible smooth representation of $W_{F}$,

(2) for any irreducible smooth representation $\sigma$ of $W_{F}$, there is an unramified character $\chi$ of $W_{F}$ such that $\chi \otimes \sigma \simeq \tau \circ \iota_{F}$, for some irreducible smooth representation $\tau$ of $\Gamma_{F}$.

2.2. The $L$-group. Let $G$ be the group of $F$-points of a connected reductive algebraic group $\mathbf{G}$ defined over $F$. Let $\mathbf{T}$ be a maximal torus of $\mathbf{G}$ defined over $F$, and let $X(\mathbf{T}):=\operatorname{Hom}_{F}\left(\mathbf{T}, \mathbb{G}_{m}\right)$ be the group of $F$-rational characters of $\mathbf{T}$. We set $X^{\vee}(\mathbf{T}):=\operatorname{Hom}_{F}\left(\mathbb{G}_{m}, \mathbf{T}\right)$. Let $R(\mathbf{G}, \mathbf{T})$ and $R^{\vee}(\mathbf{G}, \mathbf{T})$ denote the sets of roots and coroots of $\mathbf{G}$ with respect to $\mathbf{T}$, respectively. The corresponding root datum for $\mathbf{G}$ is

$$
\mathcal{R}(\mathbf{G}):=\left(X(\mathbf{T}), R(\mathbf{G}, \mathbf{T}), X^{\vee}(\mathbf{T}), R(\mathbf{G}, \mathbf{T})^{\vee}\right) .
$$

Definition 2.1. The Langlands dual group of $\mathbf{G}$ is the denote the reductive connected algebraic group $\mathbf{G}^{\vee}$, defined over $\mathbb{C}$, whose roots (resp. coroots) are the coroots (resp. roots) of $\mathbf{G}$, that is, a root datum for $\mathbf{G}^{\vee}$ is

$$
\mathcal{R}\left(\mathbf{G}^{\vee}\right):=\left(X^{\vee}(\mathbf{T}), R^{\vee}(\mathbf{G}, \mathbf{T}), X(\mathbf{T}), R(\mathbf{G}, \mathbf{T})\right) .
$$

We denote by $G^{\vee}$ the $\mathbb{C}$-points of the group $\mathbf{G}^{\vee}$.

Examples 2.2. We have

- $\mathrm{GL}_{n}(F)^{\vee}=\mathrm{GL}_{n}(\mathbb{C}), \mathrm{SL}_{n}(F)^{\vee}=\mathrm{PGL}_{n}(\mathbb{C})$, and $\mathrm{PGL}_{n}(F)^{\vee}=\mathrm{SL}_{n}(\mathbb{C})$;

- $\mathrm{Sp}_{2 n}(F)^{\vee}=\mathrm{SO}_{2 n+1}(\mathbb{C}), \mathrm{SO}_{2 n+1}(F)^{\vee}=\mathrm{Sp}_{2 n}(\mathbb{C})$;

- $\mathrm{SO}_{2 n}(F)^{\vee}=\mathrm{SO}_{2 n}(\mathbb{C})$;

- if $\mathbf{G}$ a group of exceptional type, then $\mathbf{G}$ and $\mathbf{G}^{\vee}$ are of the same type (e.g. $\left.\mathrm{G}_{2}(F)^{\vee}=\mathrm{G}_{2}(\mathbb{C})\right)$.

Of special importance is the group $\mathbf{G}=\mathrm{GL}_{n}$. Indeed, any complex reductive group $\mathbf{G}$ may be embedded into $\mathrm{GL}_{n}(\mathbb{C})$ for some $n$.

We choose a Borel subgroup $\mathbf{B} \supset \mathbf{T}$ of $\mathbf{G}$ defined over $F$, and let $\Delta$ and $\Delta^{\vee}$ denote the corresponding basis of $R(\mathbf{G}, \mathbf{T})$ and $R^{\vee}(\mathbf{G}, \mathbf{T})$. A based root datum for $\mathbf{G}$ is $\mathcal{R}_{0}(\mathbf{G}):=\left(X(\mathbf{T}), \Delta, X^{\vee}(\mathbf{T}), \Delta^{\vee}\right)$. We coose a pinning $\left(G, B, T,\left\{x_{\alpha}\right\}_{\alpha} \in \Delta\right)$, which induces a splitting of the exact sequence

$$
1 \longrightarrow \operatorname{Int}(\mathbf{G}) \longrightarrow \operatorname{Aut}(G) \longrightarrow \operatorname{Aut}\left(\mathcal{R}_{0}(G)\right) \longrightarrow 1 \text {. }
$$

The $F$ structure of $\mathbf{G}$ is given by a morphism of $\theta: \Gamma_{F} \longrightarrow \operatorname{Aut}(\mathbf{G})$ which descends to $\theta: \Gamma_{F} \longrightarrow \operatorname{Aut}\left(\mathcal{R}_{0}(\mathbf{G})\right)$.

To obtain the $L$-group as a group that actually sees $G=\mathbf{G}(F)$ and not just $\mathbf{G}$, we have to work in the Galois action of $\Gamma_{F}$ on the group $\mathbf{G}^{\vee}$. Since $\operatorname{Aut}\left(\mathcal{R}_{0}(\mathbf{G})\right) \simeq$ $\operatorname{Aut}\left(\mathcal{R}_{0}(\mathbf{G})^{\vee}\right) \simeq \operatorname{Aut}\left(\mathcal{R}_{0}\left(\mathbf{G}^{\vee}\right)\right)$, we have

$$
\theta: \Gamma_{F} \longrightarrow \operatorname{Aut}\left(\mathcal{R}_{0}(\mathbf{G})\right) \longrightarrow \operatorname{Aut}\left(\mathbf{G}^{\vee}\right) .
$$

Definition 2.3. The (Weil form of) $L$-group of $G$ is the group

$$
{ }^{L} G:=G^{\vee} \rtimes_{\theta} W_{F} .
$$


Remark 2.4. In the case when $G$ is $F$-split, we have a direct product, that is: ${ }^{L} G=G^{\vee} \times W_{F}$.

Definition 2.5. Let $\mathbf{G}, \mathbf{H}$ be two connected reductive algebraic groups over $F$. An homomorphism $\eta:{ }^{L} H \rightarrow{ }^{L} G$ is called an L-homomorphism if

- $\eta$ is continuous;

- the diagram ${ }^{L} H \longrightarrow{ }^{L} G$ commutes;

- the restriction of $\eta$ to $H^{\vee}$ is morphism of algebraic groups from $H^{\vee}$ to $G^{\vee}$.

General connected reductive $F$-groups need not be quasi-split, but they are always forms of split $F$-groups.

Definition 2.6. Two $F$-groups $G=\mathbf{G}(F)$ and $H=\mathbf{H}(F)$ are called forms of each other if $\mathbf{G}$ is isomorphic to $\mathbf{H}$ as algebraic groups, or equivalently if $\mathbf{G}\left(F_{\text {sep }}\right) \cong$ $\mathbf{H}\left(F_{\text {sep }}\right)$ as $F_{\text {sep-groups. }}$ -

An isomorphism $\vartheta: \mathbf{H} \rightarrow \mathbf{G}$ determines a 1-cocycle

$$
\gamma_{\vartheta}: \begin{array}{ccc}
\Gamma_{F} & \rightarrow \operatorname{Aut}(\mathbf{G}) \\
\sigma & \mapsto \vartheta \sigma \alpha^{-1} \sigma^{-1} .
\end{array}
$$

From $\gamma_{\vartheta}$ one can recover $H$ (up to isomorphism) as

$$
H \cong\left\{g \in \mathbf{G}\left(F_{\text {sep }}\right):\left(\gamma_{\vartheta}(\sigma) \circ \sigma\right) g=g \quad \forall \sigma \in \operatorname{Gal}\left(F_{\text {sep }} / F\right)\right\} .
$$

Given another form $\vartheta^{\prime}: \mathbf{H}^{\prime} \rightarrow \mathbf{G}$, the groups $H$ and $H^{\prime}$ are $F$-isomorphic if and only if the 1-cocycles $\gamma_{\vartheta}$ and $\gamma_{\vartheta^{\prime}}$ are cohomologous, that is, if there exists a $f \in \operatorname{Aut}(\mathbf{G})$ such that

$$
\gamma_{\vartheta}(\sigma)=f^{-1} \gamma_{\vartheta^{\prime}}(\sigma) \sigma f \sigma^{-1} \quad \forall \sigma \in \operatorname{Gal}\left(F_{\mathrm{sep}} / F\right) .
$$

In this way the isomorphism classes of forms of $G$ are in bijection with the Galois cohomology group $H^{1}(F, \operatorname{Aut}(\mathbf{G}))$.

Definition 2.7. An $F$-group $H$ is an inner form of $G$ if the cocycle $\gamma_{\vartheta}$ takes values in the group of inner automorphisms $\operatorname{Inn}(\mathbf{G})$. On the other hand, if the values of $\gamma_{\vartheta}$ are not contained in $\operatorname{Inn}(\mathbf{G})$, then $H$ is called an outer form of $G$.

Proposition 2.8. [Spr, §16.4] Every connected reductive F-group $G$ is an inner form of a unique quasi-split F-group $G^{*}$.

Example 2.9. Let $D$ be a division algebra with center $F$, of dimension $d^{2}$ over $F$. Then $G=\mathrm{GL}_{m}(D)$ is an inner form of $\mathrm{GL}_{n}(F)=G^{*}$ where $n=d m$. There is a reduced norm map Nrd: $\mathrm{GL}_{m}(D) \rightarrow F^{\times}$and the derived group $\mathrm{SL}_{m}(D):=$ $\operatorname{ker}\left(\mathrm{Nrd}: \mathrm{GL}_{m}(D) \rightarrow F^{\times}\right)$is an inner form of $\mathrm{SL}_{n}(F)$. Every inner form of $\mathrm{GL}_{n}(F)$ or $\mathrm{SL}_{n}(F)$ is isomorphic to one of this kind.

When $n=2$, the only possibilities for $d$ are 1 or 2 , and so the inner forms are, up to isomorphism, $\mathrm{GL}_{2}(F)$ and $D^{\times}$, and $\mathrm{SL}_{2}(F)$ and $\mathrm{SL}_{1}(D)$.

Definition 2.10. An inner twist of $G$ consists of a pair $(H, \vartheta)$ as above, where $H=\mathbf{H}(F)$ and $\vartheta: \mathbf{H} \stackrel{\sim}{\longrightarrow} \mathbf{G}$ are such that $\operatorname{im}\left(\gamma_{\vartheta}\right) \subset \mathbf{G}_{\mathrm{ad}}$.

Two inner twists of $G$ are equivalent if $(8)$ holds for some $f \in \operatorname{Inn}(\mathbf{G})$. Let $\operatorname{IT}(G)$ denote the set of equivalence classes of inner twists of $G$. 
Remark 2.11. It is quite possible that two inequivalent inner twists $(H, \vartheta)$ and $\left(H^{\prime}, \vartheta^{\prime}\right)$ share the same group $H \cong H^{\prime}$. This happens precisely when $\gamma_{\vartheta}$ and $\gamma_{\vartheta}$ are in the same orbit of $\operatorname{Aut}(\mathbf{G}) / \operatorname{Inn}(\mathbf{G})$ on $H^{1}\left(F, \mathbf{G}_{\mathrm{ad}}\right)$.

Kottwitz has found an important alternative description of $H^{1}(F, \mathbf{G})$. Recall that the complex dual group $G^{\vee}=\mathbf{G}^{\vee}(\mathbb{C})$ is endowed with an action of $\operatorname{Gal}\left(F_{\text {sep }} / F\right)$. There exists a natural isomorphism

$$
\kappa_{G}: H^{1}(F, \mathbf{G}) \stackrel{\sim}{\longrightarrow} \operatorname{Irr}\left(\pi_{0}\left(\mathrm{Z}\left(G^{\vee}\right)^{W_{F}}\right)\right),
$$

see [Ko, Proposition 6.4]. This is particularly useful in the following way. An inner twist of $G$ is the same thing as an inner twist of the unique quasi-split inner form $G^{*}=\mathbf{G}^{*}(F)$. Let $G_{\mathrm{ad}}^{*}=\mathbf{G}_{\mathrm{ad}}^{*}(F)$ be the adjoint group of $G^{*}$. Let $\mathbf{G}_{\mathrm{sc}}^{\vee}$ be the simply connected cover of the derived group $G_{\text {der }}^{\vee}$ of $\mathbf{G}^{\vee}$. We have $G_{\mathrm{sc}}^{\vee}=\left(G_{\text {ad }}\right)^{\vee}$, and

$$
\kappa_{G_{\mathrm{ad}}^{*}}: H^{1}\left(F, \mathbf{G}_{\mathrm{ad}}^{*}\right) \stackrel{\sim}{\longrightarrow} \operatorname{Irr}\left(\mathrm{Z}\left(G_{\mathrm{sc}}^{\vee}\right)^{W_{F}}\right) .
$$

The equivalence classes of inner twists of $G$ are parametrized by the Galois cohomology group $H^{1}\left(F, \mathbf{G}_{\text {ad }}^{*}\right)$. For every $\vartheta \in H^{1}\left(F, \mathbf{G}_{\text {ad }}^{*}\right)$, we will denote by $G_{\vartheta}$ an inner twist of $G^{*}$ which is parametrized by $\vartheta$, and we will take $G^{*}$ to be $G_{1}$.

All the inner twists of a given group $G$ share the same $L$-group, because the action of $W_{F}$ on $G^{\vee}$ is only uniquely defined up to inner automorphisms. This also works the other way round: from the Langlands dual group ${ }^{L} G$ one can recover the inner form class of $G$. Hence it is natural from the point of view of the Langlands to consider all the inner twists of a given group $G$ simultaneously.

2.3. Definitions for Langlands parameters. We write $W_{F}^{\prime}:=W_{F} \times \mathrm{SL}_{2}(\mathbb{C})$ (that may be viewed as version of the Weil-Deligne group of $F$ ).

Definition 2.12. A Langlands parameter (or $L$-parameter, for short) for ${ }^{L} G$ is a smooth morphism

$$
\phi: W_{F}^{\prime} \rightarrow{ }^{L} G
$$

such that $W_{F}^{\prime} \longrightarrow{ }^{L} G$ commutes, $\phi(w)$ is semisimple for each $w \in W_{F}$

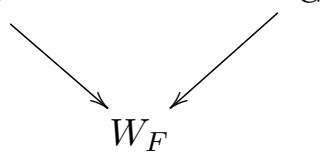

(that is, $r(\phi(w))$ is semisimple for every finite dimensional representation $r$ of ${ }^{L} G$ ), and $\left.\phi\right|_{\mathrm{SL}_{2}(\mathbb{C})}$ is a morphism of complex algebraic groups.

The group $G^{\vee}$ acts on the set $\Phi\left({ }^{L} G\right)$ of such $\phi$ 's by conjugation. Let $\Phi\left({ }^{L} G\right)$ denote the set of $G^{\vee}$-orbits in $\underline{\Phi}\left({ }^{L} G\right)$

Definition 2.13. An $L$-parameter $\phi: W_{F}^{\prime} \rightarrow{ }^{L} G$ is said to be

- unramified if it has trivial restriction to $I_{F}$;

- tame if it has trivial restriction to the wild ramification group $P_{F}$ of $F$.;

- essentially tame if $\phi\left(P_{F}\right)$ lies in a maximal torus of $G^{\vee}$;

- discrete (or elliptic) if there is no proper $W_{F}$-stable Levi subgroup $L^{\vee} \subset G^{\vee}$ such that $\phi\left(W_{F}^{\prime}\right) \subset{ }^{L} L$.

- bounded if $\phi^{\prime}\left(W_{F}\right) \subset G^{\vee}$ is bounded, where $\phi(w)=\left(\phi^{\prime}(w), w\right)$. (This is equivalent to $\phi^{\prime}$ (Frob) being a compact element of $G^{\vee}$. 
2.3.1. Relevance for Langlands parameters. We will call a Levi factor of a parabolic subgroup of $G$ simply a Levi subgroup of $G$. The bijection

$$
R(\mathbf{G}, \mathbf{T}) \longleftrightarrow R^{\vee}(\mathbf{G}, \mathbf{T})=R\left(G^{\vee}, T^{\vee}\right)
$$

gives a basis $\Delta^{\vee}$, and provides a canonical bijection between the sets of conjugacy classes of parabolic subgroups of $\mathbf{G}$ and of $G^{\vee}$.

Definition 2.14. [Bor, $\S 3$ ] A parabolic subgroup $P^{\vee}$ of $G^{\vee}$ is F-relevant if the corresponding class of parabolic subgroups of $\mathbf{G}$ contains an element $\mathbf{P}$ which is defined over $F$. Similarly, a Levi subgroup $L^{\vee} \subset G^{\vee}$ is $F$-relevant if it is a Levi factor of a parabolic subgroup $P^{\vee} \subset G^{\vee}$ which is $F$-relevant.

Definition 2.15. A parabolic subgroup $P^{\vee}$ of $G^{\vee}$ is $W_{F}$-quasi-stable if the projection $\mathrm{N}_{L_{G}}\left(P^{\vee}\right) \rightarrow W_{F}$ is surjective.

Remark 2.16. The $W_{F}$-quasi-stable parabolic subgroups of $G^{\vee}$ are precisely the neutral components of what Borel [Bor, §3] calls parabolic subgroups of ${ }^{L} G$.

Definition 2.17. Let $\left.\phi \in \underline{\Phi}\left({ }^{L} G\right)\right)$ and let $P^{\vee}$ be a $W_{F}$-quasi-stable parabolic subgroup of $G^{\vee}$ with a Levi factor $L^{\vee}$ such that

- the image of $\phi$ is contained in $\mathrm{N}_{L_{P}}\left(L^{\vee}\right)$, where ${ }^{L} P:=P^{\vee} \rtimes W_{F}$;

- $P^{\vee}$ is a minimal for this property.

The $L$-parameter $\phi$ is $G$-relevant (or is an $L$-parameter for $G$ ) if $P^{\vee}$ is $F$-relevant.

Notation 2.18. We denote by $\Phi(G)$ the subset of $\Phi\left({ }^{L} G\right)$ of the $\mathbf{G}^{\vee}$-conjugacy classes of $L$-parameters that are $G$-relevant.

A Langlands classification of $L$-parameters for $G$ is obtained in [SZ].

We have

$$
\Phi\left({ }^{L} G\right)=\Phi\left(G^{*}\right),
$$

where $G^{*}$ is the quasi-split inner form of $G$ as defined in Proposition 2.8, and it is expected that in general $\Pi_{\phi}(G)$ is nonempty if and only if $\phi$ is $G$-relevant.

Example 2.19. Let $D$ be a division algebra with center $F$ over $F$ such that $\operatorname{dim}_{F}(D)=4$. Then $G=D^{\times}$is the unique non-split inner form of $G^{*}=\mathrm{GL}_{2}(F)$.

The only Levi subgroup of $D^{\times}$defined over $F$ is $D^{\times}$itself, and it corresponds to the Levi subgroup $\mathrm{GL}_{2}(\mathbb{C})$ on the complex side.

Let $\phi \in \underline{\Phi}\left(\mathrm{GL}_{2}(F)\right)=\underline{\Phi}\left(D^{\times}\right)$be the embedding $W_{F}^{\prime} \hookrightarrow \mathrm{GL}_{2}(\mathbb{C}) \times W_{F}$. No proper parabolic subgroup of $\mathrm{GL}_{2}(\mathbb{C})$ contains $\phi\left(\mathrm{SL}_{2}(\mathbb{C})\right)=\mathrm{SL}_{2}(\mathbb{C})$, so $\phi$ is relevant for both $D^{\times}$and $\mathrm{GL}_{2}(F)$.

\subsection{L-packets.}

2.4.1. For a given group $G$. The local Langlands conjecture (LLC) asserts that the set $\operatorname{Irr}(G)$ of isomorphism classes of irreducible smooth representations of $G$ can be parametrized by the set $\Phi(G)$. This parametrization is usually not a bijection. In fact, it is conjectured that each conjugacy class $\phi \in \Phi(G)$ is associated with a finite set $\Pi_{\phi}(G)$ of isomorphism classes of irreducible smooth representations of $G$, and that they give a disjoint decomposition of $\operatorname{Irr}(G)$;

$$
\operatorname{Irr}(G)=\bigsqcup_{\phi \in \Phi(G)} \Pi_{\phi}(G)
$$


Such finite sets are called L-packets for $G$. This parametrization is based on the belief that there should be certain arithmetic invariants (e.g., $L$-factors) defined on both the representation side and the parameter side so that one could match them. From this point of view, one can think that the $L$-packet $\Pi_{\phi}$ attached to some $\phi \in \Phi(G)$ consists of all irreducible smooth representations of $G$ whose arithmetic invariants match those of $\phi$.

2.4.2. For all the inner twists of $G$. The local Langlands correspondence predicts the existence of a partition of the set $\operatorname{Irr}(\operatorname{IT}(G)$ of equivalence classes of the irreducible smooth representations of all the groups $G_{i}$ in $\operatorname{IT}(G)$ into finite subsets:

$$
\operatorname{Irr}(\operatorname{IT}(G))=\bigsqcup_{\phi \in \Phi\left({ }^{L} G\right)} \Pi_{\phi}\left({ }^{L} G\right),
$$

where each $L$-packet $\Pi_{\phi}\left({ }^{L} G\right)$ is the union of $L$-packets for the groups $G_{i}$.

Example 2.20. We keep the notation of Example 2.19. We have

$$
\Pi_{\phi}\left(\mathrm{GL}_{2}(F)\right)=\left\{\mathrm{St}_{\mathrm{GL}_{2}(F)}\right\} \quad \text { and } \quad \Pi_{\phi}\left(D^{\times}\right)=\left\{\operatorname{St}_{D^{\times}}=\operatorname{triv}_{D^{\times}}\right\} .
$$

Thus here (11) is

$$
\Pi_{\phi}\left({ }^{L} G\right)=\Pi_{\phi}\left(\mathrm{GL}_{2}(F)\right) \cup \Pi_{\phi}\left(D^{\times}\right)=\left\{\mathrm{St}_{\mathrm{GL}_{2}(F)}, \mathrm{St}_{D^{\times}}\right\} .
$$

Let $\phi^{\prime} \in \underline{\Phi}\left(\mathrm{GL}_{2}(\mathbb{C})\right)$ such that $\phi^{\prime}\left(\mathrm{SL}_{2}(\mathbb{C})\right)=1$ and $\phi^{\prime}\left(W_{F}\right) \subset \operatorname{diag}\left(\mathrm{GL}_{2}(\mathbb{C})\right) \times W_{F}$. Then $L^{\vee}=\operatorname{diag}\left(\mathrm{GL}_{2}(\mathbb{C})\right)$ is the minimal Levi subgroup such that ${ }^{L} L$ contains the image of $\phi_{2}$. Thus the standard Borel subgroup $P^{\vee}$ of $\mathrm{GL}_{2}(\mathbb{C})$ satisfies the conditions in Definition 2.17. But its conjugacy class does not correspond to any parabolic subgroup of $D^{\times}$, so $\phi^{\prime}$ is not relevant for $D^{\times}$.

Let $\mathbf{G} \subset \widetilde{\mathbf{G}}$ be a pair of quasi-split connected reductive groups, defined over $F$, with the same derived group. It is expected that the $L$-packets of $G=\mathbf{G}(F)$ are restrictions of $L$-packets of $\widetilde{G}:=\widetilde{\mathbf{G}}(F)$ in the sense that for each $L$-packet $\Pi(G)$ for $G$ there is a packet $\Pi(\widetilde{G})$ for $\widetilde{G}$ whose restriction to $G$ is equal to $\Pi$. Typically one uses this last expectation to construct $L$-packets for $G$ from the knowledge of $L$-packets of $\widetilde{G}$, e.g., in the cases of $\mathrm{SL}_{n} \subset \mathrm{GL}_{n}$ and $\mathrm{Sp}_{2 n} \subset \mathrm{GSp}_{2 n}$. In some cases, however, we wish to move in the other direction, and use the knowledge of $L$-packets of $G$ to obtain structural information about the $L$-packets of $\widetilde{G}$, see $[\mathrm{Xu}]$.

\section{ENHANCED LANGLANDS PARAMETERS}

3.1. The group $\mathcal{S}_{\phi}$. To parametrize the irreducible representations in a given $L$ packet, we need more information then just the Langlands parameter itself. Let $\mathbf{Z}_{\mathbf{G}^{\vee}}(\phi)$ denote the centralizer of $\phi\left(W_{F}^{\prime}\right)$ in $\mathbf{G}^{\vee}$. This is a reductive algebraic group over $\mathbb{C}$, in general disconnected. We denote by $\mathbf{Z}_{\mathbf{G}^{\vee}}$ the center of $\mathbf{G}^{\vee}$ and we write

$$
\mathcal{R}_{\phi}:=\pi_{0}\left(\mathrm{Z}_{\mathbf{G}} \vee(\phi) / \mathrm{Z}\left(\mathbf{G}^{\vee}\right)^{W_{F}}\right) .
$$

Remark 3.1. It is expected that $\Pi_{\phi}(G)$ is in bijection with $\operatorname{Irr}\left(\mathcal{R}_{\phi}\right)$ if $G$ is quasisplit. However, for $G$ non necessarily quasi-split, this is not always the case. $\left.\mathbf{G}^{\vee}\right)$.

Let $\mathbf{G}_{a d}^{\vee}$ be the quotient $\mathbf{G}^{\vee} / \mathbf{Z}_{\mathbf{G}^{\vee}}$ (that is, the group $\mathbf{G}_{a d}^{\vee}$ is the adjoint group of 
Since $\mathbf{Z}_{\mathbf{G}^{\vee}}(\phi) \cap \mathrm{Z}\left(\mathbf{G}^{\vee}\right)=\mathrm{Z}\left(\mathbf{G}^{\vee}\right)^{W_{F}}$,

$$
\mathrm{Z}_{\mathbf{G}^{\vee}}(\phi) / \mathrm{Z}\left(\mathbf{G}^{\vee}\right)^{W_{F}} \cong \mathrm{Z}_{\mathbf{G}^{\vee}}(\phi) \mathrm{Z}\left(\mathbf{G}^{\vee}\right) / \mathrm{Z}\left(\mathbf{G}^{\vee}\right) .
$$

The right hand side can be considered as a subgroup of the adjoint group $\mathbf{G}_{\mathrm{ad}}^{\vee}$. Let $\mathrm{Z}_{\mathbf{G}_{\mathrm{sc}}^{\vee}}^{1}(\phi)$ be its inverse image under the quotient map $\mathbf{G}_{\mathrm{sc}}^{\vee} \rightarrow \mathbf{G}_{\mathrm{ad}}^{\vee}$. We can also characterize it as

$$
\begin{array}{r}
=\left\{g \in \mathrm{Z}_{\mathbf{G}_{\mathrm{sc}}^{\vee}}\left(\phi\left(\mathrm{SL}_{2}(\mathbb{C})\right)\right):\left.g \phi\right|_{W_{F}} g^{-1}=\left.\phi\right|_{W_{F}} a_{g} \text { for some } a_{g} \in B^{1}\left(W_{F}, \mathrm{Z}\left(\mathbf{G}^{\vee}\right)\right)\right\} \\
=\mathrm{Z}_{\mathbf{G}_{\mathrm{sc}}^{\vee}}^{1}\left(\left.\phi\right|_{W_{F}}\right) \cap \mathrm{Z}_{\mathbf{G}_{\mathrm{sc}}^{\vee}}\left(\phi\left(\mathrm{SL}_{2}(\mathbb{C})\right)\right),
\end{array}
$$

where $B^{1}\left(W_{F}, \mathrm{Z}\left(\mathbf{G}^{\vee}\right)\right)$ denotes the set of 1-coboundaries for group cohomology, that is, the maps $W_{F} \rightarrow \mathrm{Z}\left(\mathbf{G}^{\vee}\right)$ of the form $w \mapsto z w z^{-1} w^{-1}$ with $z \in \mathbf{Z}\left(\mathbf{G}^{\vee}\right)$. The neutral component of $\mathbf{Z}_{\mathbf{G}_{\mathrm{sc}}^{\vee}}^{1}(\phi)$ is $\mathbf{Z}_{\mathbf{G}_{\mathrm{sc}}^{\vee}}(\phi)^{\circ}$, so it is a complex reductive group.

Remark 3.2. We have $\mathrm{Z}_{\mathbf{G}_{\mathrm{sc}}^{\vee}}^{1}(\phi)=\mathrm{Z}_{\mathbf{G}_{\mathrm{sc}}^{\vee}}(\phi)$ whenever $\mathrm{Z}\left(\mathbf{G}_{\mathrm{sc}}^{\vee}\right)^{W_{F}}=\mathrm{Z}\left(\mathbf{G}_{\mathrm{sc}}^{\vee}\right)$, in particular if $G$ is an inner twist of a split group.

Following Arthur Ar1], given $\phi$, we define the group $\mathcal{S}_{\phi}$ as the component group of $\mathrm{Z}_{\mathbf{G} \vee}^{1}(\phi)$ :

$$
\mathcal{S}_{\phi}:=\pi_{0}\left(\mathrm{Z}_{\mathbf{G}_{\mathrm{sc}}^{\vee}}^{1}(\phi)\right) .
$$

Via (13), the map $\mathbf{G}_{\mathrm{sc}}^{\vee} \rightarrow \mathbf{G}_{\text {ad }}^{\vee}$ induces a homomorphism $\mathcal{S}_{\phi} \rightarrow \mathcal{R}_{\phi}$. We set

$$
\mathcal{Z}_{\phi}:=\mathrm{Z}\left(\mathbf{G}_{\mathrm{sc}}^{\vee}\right) / \mathrm{Z}\left(\mathbf{G}_{\mathrm{sc}}^{\vee}\right) \cap \mathrm{Z}_{\mathbf{G}_{\mathrm{sc}}^{\vee}}(\phi)^{\circ}
$$

. Then (see [ABPS7, Lemma 1.7]) $\mathcal{S}_{\phi}$ is a central extension of $\mathcal{R}_{\phi}$ by $\mathcal{Z}_{\phi}$ :

$$
1 \rightarrow \mathcal{Z}_{\phi} \rightarrow \mathcal{S}_{\phi} \rightarrow \mathcal{R}_{\phi} \rightarrow 1 \text {. }
$$

Since $\mathbf{G}_{\mathrm{sc}}^{\vee}$ is a central extension of $\mathbf{G}_{\mathrm{ad}}^{\vee}=\mathbf{G}^{\vee} / \mathrm{Z}\left(\mathbf{G}^{\vee}\right)$, the conjugation action of $\mathbf{G}_{\mathrm{sc}}^{\vee}$ on itself and on $\mathcal{S}_{\phi}$ descends to an action of $\mathbf{G}_{\mathrm{ad}}^{\vee}$. Via the canonical quotient map, also $\mathbf{G}^{\vee}$ acts on $\mathcal{S}_{\phi}$ by conjugation.

We attach to a given $L$-parameter $\phi \in \underline{\Phi}\left({ }^{L} G\right)$ the following (possibly disconnected) complex reductive group:

$$
\mathcal{G}_{\phi}:=\mathrm{Z}_{\mathbf{G}_{\mathrm{sc}}^{\vee}}^{1}\left(\phi\left(W_{F}\right)\right) .
$$

The following proposition provides a way to link the local Langlands correspondence for $G$ to the generalized Springer correspondence for the groups $\mathcal{G}_{\phi}$ 's.

Proposition 3.3. AMS1, (92)] The group $\mathcal{S}_{\phi}$ is isomorphic to the group

$$
A_{\mathcal{G}_{\phi}}\left(u_{\phi}\right):=\pi_{0}\left(\mathrm{Z}_{\mathcal{G}_{\phi}}\left(u_{\phi}\right)\right) \text {, }
$$

where $u_{\phi}:=\phi\left(1,\left(\begin{array}{ll}1 & 1 \\ 0 & 1\end{array}\right)\right)$

\subsection{Definition of enhanced Langlands parameters.}

Definition 3.4. An enhanced Langlands parameter (or enhanced $L$-parameter) for ${ }^{L} G$ is a pair $(\phi, \rho)$, where $\phi \in \underline{\Phi}\left({ }^{L} G\right)$ and $\rho$ is an irreducible representation of the group $\mathcal{S}_{\phi}$ defined in 15 .

We let $\mathbf{G}^{\vee}$ and $\mathbf{G}_{\mathrm{sc}}^{\vee}$ act on the set of enhanced $L$-parameters for ${ }^{L} G$ by

$$
g \cdot(\phi, \rho)=\left(g \phi g^{-1}, g \cdot \rho\right) \quad \text { where } \quad(g \cdot \rho)(a)=\rho\left(g^{-1} a g\right) .
$$

We note that both groups acting in 19 yield the same orbit space. 
Notation 3.5. Let $\Phi_{\mathrm{e}}\left({ }^{L} G\right)$ denote the set of $\mathbf{G}^{\vee}$-conjugacy classes of enhanced Langlands parameters for ${ }^{L} G$.

We set

$$
\mathcal{Z}_{\phi}^{W_{F}}:=\mathrm{Z}\left(G_{\mathrm{sc}}^{\vee}\right)^{W_{F}} /\left(\mathrm{Z}\left(G_{\mathrm{sc}}^{\vee}\right)^{W_{F}} \cap Z_{G_{\mathrm{sc}}^{\vee}}(\phi)^{\circ}\right) .
$$

According to [Ar1, §4]

$$
\mathrm{Z}\left(G_{\mathrm{sc}}^{\vee}\right) \cap \mathrm{Z}_{G_{\mathrm{sc}}^{\vee}}(\phi)^{\circ} \subset \mathrm{Z}\left(G_{\mathrm{sc}}^{\vee}\right)^{W_{F}}
$$

Hence $\mathcal{Z}_{\phi}^{W_{F}}$ can be regarded as a subgroup of $\mathcal{Z}_{\phi}$ and

$$
\mathcal{Z}_{\phi} / \mathcal{Z}_{\phi}^{W_{F}} \cong \mathrm{Z}\left(G_{\mathrm{sc}}^{\vee}\right) / \mathrm{Z}\left(G_{\mathrm{sc}}^{\vee}\right)^{W_{F}}
$$

By Schur's lemma every enhanced Langlands parameter $(\phi, \rho)$ restricts to a character $\left.\rho\right|_{\mathcal{Z}_{\phi}^{W_{F}}}$ of $\mathcal{Z}_{\phi}^{W_{F}}$. This can be inflated to a character $\zeta_{\rho}$ of $\mathrm{Z}\left(\mathbf{G}_{\mathrm{sc}}^{\vee}\right)^{W_{F}}$. With the Kottwitz isomorphism (9) we get an element $\vartheta:=\kappa_{G_{\text {ad }}^{*}}^{-1}\left(\zeta_{\rho}\right) \in H^{1}\left(F, \mathbf{G}_{\mathrm{ad}}^{*}\right)$. In this way $(\phi, \rho)$ determines a unique inner twist $G_{\vartheta}$ of $G$. This can be regarded as an alternative way to specify for which inner twists of $G$ an enhanced Langlands parameter is relevant. Fortunately, it turns out that it agrees with the earlier definition of relevance of Langlands parameters.

Indeed for $\phi \in \Phi\left({ }^{L} G\right)$ the following are equivalent (see [ABPS7, Prop. 1.8])

(1) $\phi$ is relevant for $G_{\vartheta}$;

(2) $Z\left(G_{\mathrm{sc}}^{\vee}\right)^{W_{F}} \cap \mathrm{Z}_{G_{\mathrm{sc}}^{\vee}}(\phi)^{\circ} \subset \operatorname{ker} \zeta$;

(3) there exists a $\rho \in \operatorname{Irr}\left(\mathcal{S}_{\phi}\right)$ such that $\zeta$ is the lift of $\left.\rho\right|_{\mathcal{Z}_{\phi}^{W_{F}}}$ to $\mathrm{Z}\left(G_{\mathrm{sc}}^{\vee}\right)^{W_{F}}$.

Here $\zeta \in \operatorname{Irr}\left(\mathrm{Z}\left(G_{\mathrm{sc}}^{\vee}\right)^{W_{F}}\right)$ and $G_{\vartheta}$ is the inner twist of $G$ associated to $\vartheta=\kappa_{G_{\mathrm{ad}}^{*}}^{-1}(\zeta)$ via (9).

Notation 3.6. We denote by $\Phi_{\mathrm{e}}(G)$ the set of $\mathbf{G}^{\vee}$-equivalence classes of enhanced $L$-parameters that are $G$-relevant.

Let $(\phi, \rho) \in \Phi_{\mathrm{e}}\left({ }^{L} G\right)$. If $\rho$ is relevant for $G$, then $\zeta_{\rho} \in \operatorname{Irr}\left(\mathrm{Z}\left(G_{\mathrm{sc}}^{\vee}\right)^{W_{F}}\right)$. It can be extended in precisely $\left[\mathrm{Z}\left(G_{\mathrm{sc}}^{\vee}\right): \mathrm{Z}\left(G_{\mathrm{sc}}^{\vee}\right)^{W_{F}}\right]$ ways to a character of $\mathrm{Z}\left(G_{\mathrm{sc}}^{\vee}\right)$. We choose such an extension and we denote it by $\zeta_{G}$. Every $\phi \in \underline{\Phi}\left({ }^{L} G\right)$ can be enhanced with a $\rho \in \operatorname{Irr}\left(\mathcal{S}_{\phi}\right)$ such that $\left.\rho\right|_{\mathcal{Z}_{\phi}}$ inflates to $\zeta_{G}$.

We denote the set of equivalence classes of such $(\phi, \rho) \in \underline{\Phi}_{\mathrm{e}}\left({ }^{L} G\right)$ by $\Phi_{\mathrm{e}, \zeta_{G}}\left({ }^{L} G\right)$. Of course we pick $\zeta_{G}=\operatorname{triv}$ when $G=G^{*}$. We have

$$
\Phi_{\mathrm{e}, \zeta_{G}}\left({ }^{L} G\right)=\Phi_{\mathrm{e}}(G)
$$

and, in particular $\Phi_{\mathrm{e}, \text { triv }}\left({ }^{L} G\right)=\Phi_{\mathrm{e}}\left(G^{*}\right)$.

3.3. Desiderata for the local Langlands correspondence. We are ready to formulate the version of the conjectural local Langlands correspondence stated in [ABPS7]. It is inspired by many sources, in particular [Bor, §10], Vog, §4], [Ar1, $\S 3$ ] and [Hai, $\S 5.2$ ].

Conjecture 3.7. Let $(G, \vartheta)$ be an inner twist of a quasi-split F-group $G^{*}$. There exists a surjection

$$
\Phi_{\mathrm{e}}\left({ }^{L} G\right) \longrightarrow \operatorname{Irr}(G):(\phi, \rho) \mapsto \pi_{\phi, \rho},
$$


which becomes bijective when restricted to $\Phi_{\mathrm{e}, \zeta_{G}}(G)=\Phi_{\mathrm{e}}(G)$. We write its inverse as

$$
\operatorname{Irr}(G) \longrightarrow \Phi_{\mathrm{e}}(G): \pi \mapsto\left(\phi_{\pi}, \rho_{\pi}\right)
$$

Then the map $\operatorname{Irr}(G) \rightarrow \Phi(G): \pi \mapsto \phi_{\pi}$ is canonical. These maps satisfy the properties (1) - (7) listed below.

Remark 3.8. The above bijection becomes more elegant if one considers the union over inner twists, then it says that there exists a surjection

$\left\{(\phi, \rho): \phi \in \Phi\left(G^{*}\right), \rho \in \operatorname{Irr}\left(\mathcal{S}_{\phi}\right)\right\} \rightarrow\left\{(G, \vartheta, \pi):(G, \vartheta)\right.$ inner twist of $\left.G^{*}, \pi \in \operatorname{Irr}(G)\right\}$

whose fibers have exactly $\left[\mathrm{Z}\left(G_{\mathrm{sc}}^{\vee}\right): \mathrm{Z}\left(G_{\mathrm{sc}}^{\vee}\right)^{W_{F}}\right]$ elements.

Properties:

(1) The central character of $\pi$ equals the character of $\mathrm{Z}(G)$ constructed from $\phi_{\pi}$ in [Bor, $\S 10.1]$.

(2) Let $z \in H_{c}^{1}\left(W_{F}, \mathrm{Z}\left(\mathbf{G}^{\vee}\right)\right)$ be a class in continuous group cohomology, and let $\chi_{z}: G \rightarrow \mathbb{C}^{\times}$be the character associated to it in [Bor, §10.2]. Thus $z \phi_{\pi} \in$ $\tilde{\Phi}(G)$ and $\mathcal{S}_{z \phi_{\pi}}=\mathcal{S}_{\phi_{\pi}}$. Then the LLC should satisfy $\left(z \phi_{\pi}, \rho_{\pi}\right)=\left(\phi_{\chi_{z} \pi}, \rho_{\chi_{z} \pi}\right)$.

(3) $\pi$ is essentially square-integrable if and only if $\phi_{\pi}$ is discrete.

(4) $\pi$ is tempered if and only if $\phi_{\pi}$ is bounded.

(5) Let $P$ be a parabolic subgroup of $G$ with Levi factor $L$. Suppose that $g \in$ $\mathrm{N}_{G}(L)$ and $\check{g} \in \mathrm{N}_{G^{\vee}}\left(L^{\vee}\right)$ are such that $\operatorname{Ad}(g): L \rightarrow L$ and $\operatorname{Ad}(\check{g}): L^{\vee} \rightarrow L^{\vee}$ form a corresponding pair of homomorphisms, in the sense of [Bor, §2]. Then

$$
\left(\phi_{g \cdot \pi}, \rho_{g \cdot \pi}\right)=\left(\operatorname{Ad}(\check{g}) \phi_{\pi}, \check{g} \cdot \rho_{\pi}\right) \quad \text { for all } \quad \pi \in \operatorname{Irr}(L) .
$$

(6) Suppose that $\left(\phi^{L}, \rho^{L}\right) \in \Phi_{\mathrm{e}}(L)$ is bounded. Then

$$
\left\{\pi_{\phi, \rho}: \phi=\phi^{L} \text { composed with }{ }^{L} L \rightarrow{ }^{L} G,\left.\rho\right|_{\mathcal{S}_{\phi}^{L}} \text { contains } \rho^{L}\right\}
$$

equals the set of irreducible constituents of the parabolically induced representation $\mathrm{I}_{P}^{G}\left(\pi_{\phi^{L}, \rho^{L}}\right)$.

(7) If $\phi^{L}$ is discrete but not necessarily bounded, then (23) is the set of Langlands constituents of $\mathrm{I}_{P}^{G}\left(\pi_{\phi^{L}, \rho^{L}}\right)$, as in [ABPS1, p. 30].

3.3.1. Inner forms of $\mathrm{GL}_{n}(F)$. The local Langlands correspondence for supercuspidal representations of $\mathrm{GL}_{n}(F)$ was established first for $F$ of positive chacteristic in [LRS], and later for $F$ of characteristic zero in [HaTa], Hen2] and [Scho, independently.

Together with the Jacquet-Langlands correspondence this provides the LLC for essentially square-integrable representations of inner forms $G=\mathrm{GL}_{m}(D)$ of $\mathrm{GL}_{n}(F)$. It is extended to all irreducible $G$-representations via the Zelevinsky classification [Zel], see [HiSa, and ABPS4]. For these groups every $L$-packet is a singleton and the LLC is a canonical bijective map

$$
\operatorname{rec}_{\mathrm{GL}_{m}(D)}: \operatorname{Irr}\left(\mathrm{GL}_{m}(D)\right) \rightarrow \Phi\left(\mathrm{GL}_{m}(D)\right)
$$


3.3.2. Inner forms of $\mathrm{SL}_{n}(F)$. The local Langlands correspondence for $\mathrm{SL}_{m}(D)$ was established [HiSa] (for $F$ of characteristic zero) and ABPS4] (for $F$ of positive characteristic). It is derived from the LLC for $\mathrm{GL}_{m}(D)$, in the sense that every $L$-packet for $\mathrm{SL}_{m}(D)$ consists of the irreducible constituents of

$$
\operatorname{Res}_{\mathrm{SL}_{m}(D)}^{\mathrm{GL}_{m}(D)}\left(\Pi_{\phi}\left(\mathrm{GL}_{m}(D)\right)\right)
$$

with $\phi \in \underline{\Phi}\left(\mathrm{GL}_{m}(D)\right)$. Of course these $L$-packets have more than one element in general (as showed Example 2.20).

3.3.3. The local Langlands conjecture for tempered representation. Let $\operatorname{Irr}^{\mathrm{t}}(G)$ denote the subset of $\operatorname{Irr}(G)$ consisting of all irreducible smooth representations $\pi$ whose matrix coefficients are $L^{2+\epsilon}$ on $G$ modulo the center $\mathrm{Z}(G)$, and let $\Phi^{\mathrm{bd}}(G)$ be the subset of $\Phi(G)$ consisting of $\mathbf{G}^{\vee}$-conjugacy classes of $L$-parameters $\phi$ such that the $\phi\left(W_{F} \times \mathrm{SU}_{2}\right)$ is bounded modulo the center.

The local Langlands conjecture for tempered representation asserts the existence of a surjective map

$$
\operatorname{rec}_{G}^{\mathrm{t}}: \operatorname{Irr}^{\mathrm{t}}(G) \rightarrow \Phi^{\mathrm{bd}}(G),
$$

the fibers of it are called the tempered L-packets. For each $\phi \in \Phi^{\mathrm{bd}}(G)$, the corresponding tempered $L$-packet $\Pi_{\phi}^{\mathrm{t}}(G)$ is expected to be parametrized by $\operatorname{Irr}\left(S_{\phi}\right)_{\zeta_{G}}$, and $\operatorname{Irr}^{\mathrm{t}}(G)$ to be a disjoint union of the $\Pi_{\phi}^{\mathrm{t}}(G)$. In the case when the characteristic of $F$ is zero, this conjecture has been proved by Arthur in Ar2 for quasi-split orthogonal groups and symplectic groups, by Mok in [Mok] for quasi-split unitary groups, and by Kaletha, Minguez, Shin and White for non in [KMSW]. Note that the unitary group $\mathrm{U}_{E / F}(n)$ admits a non-quasi-split inner form exactly when $n$ is even.

The LLC was also proved for the $\operatorname{groups} \mathrm{GSp}_{4}(F)$ GaTak1] and [Ga], its inner form [GaTan], the group $\mathrm{Sp}_{4}(F)$ GaTak2] and its inner form [Ch], and for the groups $\mathrm{GSpin}_{4}, \mathrm{GSpin}_{6}$, and their inner forms [AsCh].

In GaVa, Ganapathy and Varma used Arthur's results to lift the LLC for split symplectic and special orthogonal groups on a non Archimedean field of odd positive characteristic, but using a "Gan-Takeda type" characterization instead of the theory of endoscopy. They proved (see GaVa, Theorem 13.6.1]) that given a tempered representation $\pi$ of $G$, there exists a unique bounded Langlands parameter $\phi_{\pi}$, defined by suitable compatibility conditions on Langlands-Shahidi $L$-functions and $\gamma$-factors, and on Plancherel measures, together with the requirement that $\phi_{\pi}$ is discrete if $\pi$ belongs to the discrete series.

For nice and precise states of art of the local Langlands conjecture the reader should consult [Kal2], [JN] and [Lom, the latter including global aspects and the link with the Ramanujan conjecture. A survey on other aspects of the Langlands correspondance may be found in $[\mathrm{Au}]$ and the references herein.

3.4. Depth. Another invariant that makes sense on both sides of the LLC is the depth. The depth $d(\pi)$ of an irreducible smooth representation $\pi$ of a reductive $p$-adic group $G$ was defined by Moy and Prasad $\mathrm{MoPr}$ in terms of filtrations $G_{x, r}$ (with $x$ a point in the Bruhat-Tits buiding of $G$ and $\left.r \in \mathbb{R}_{\geq 0}\right)$ of its parahoric subgroups $G_{x, 0}$. 
The depth of a Langlands parameter $\phi$ is defined to be the smallest number $d(\phi) \geq 0$ such that $\phi$ is trivial on $\Gamma^{r}$ for all $r>d(\phi)$.

$\mathrm{Yu}[\mathrm{Yu}, \S 7.10]$ proved that the depth is preserved by the LLC for unramified tori. Recently, Mishra and Patanayak proved that it is not preserved for wildly ramified tori $[\mathrm{MiPa}]$.

3.4.1. Inner forms of general linear groups. Let $\mathrm{GL}_{m}(D)$ an inner form of $\mathrm{GL}_{n}(F)$. Let $k_{D}=\mathfrak{o}_{D} / \mathfrak{p}_{D}$ be the residual field of $D$. Let $\mathfrak{A}$ be a hereditary $\mathfrak{o}_{F}$-order in $\mathrm{M}_{m}(D)$. The Jacobson radical of $\mathfrak{A}$ will be denoted by $\mathfrak{P}$. Let $r=e_{D}(\mathfrak{A})$ and $e=e_{F}(\mathfrak{A})$ denote the integers defined by $\mathfrak{p}_{D} \mathfrak{A}=\mathfrak{P}^{r}$ and $\mathfrak{p}_{F} \mathfrak{A}=\mathfrak{P}^{e}$, respectively. We have $e_{F}(\mathfrak{A})=d e_{D}(\mathfrak{A})$. The normalizer in $G$ of $\mathfrak{A}^{\times}$will be denoted by

$$
\mathfrak{K}(\mathfrak{A}):=\left\{g \in G: g^{-1} \mathfrak{A}^{\times} g=\mathfrak{A}^{\times}\right\} .
$$

Define a sequence of compact open subgroups of $G=\mathrm{GL}_{m}(D)$ by

$$
U^{0}(\mathfrak{A}):=\mathfrak{A}^{\times}, \quad \text { and } \quad U^{j}(\mathfrak{A}):=1+\mathfrak{P}^{j}, \quad j \geq 1 .
$$

Then $\mathfrak{A}^{\times}$is a parahoric subgroup of $G$ and $U^{1}(\mathfrak{A})$ is its pro-unipotent radical. We define the normalized level of an irreducible representation $\pi$ of $G$ to be

$$
\ell(\pi):=\min \left\{j / e_{F}(\mathfrak{A})\right\},
$$

where $(j, \mathfrak{A})$ ranges over all pairs consisting of an integer $j \geq 0$ and a hereditary $\mathfrak{o}_{F}$-order $\mathfrak{A}$ in $\mathrm{M}_{m}(D)$ such that $\pi$ contains the trivial character of $U^{j+1}(\mathfrak{A})$. Then (see for instance [ABPS3, Proposition 2.5]) the normalized level of $\pi \in \operatorname{Irr}(G)$ equals its Moy-Prasad depth:

$$
\ell(\pi)=d(\pi)
$$

Let $\psi$ be a nontrivial character of $F$ and let $c(\psi)$ be the largest integer $c$ such that $\mathfrak{p}_{F}^{-c} \subset \operatorname{ker} \psi$. The $\epsilon$ factor of $\phi($ and $\psi)$ was defined in [Tat]. It takes the form

$$
\epsilon(s, \phi, \psi)=\epsilon(0, \phi, \psi) q^{-(a(\phi)+n c(\psi)) s} \text { with } \epsilon(0, \phi, \psi) \in \mathbb{C}^{\times} .
$$

Here $a(\phi) \in \mathbb{Z}_{\geq 0}$ is the Artin conductor of $\phi$ (called $f(\phi)$ in [Ser, $\S$ VI.2]). For any elliptic $\phi \in \Phi\left(\bar{G} L_{n}(F)\right)$, we have (see [ABPS3, Lemma 2.3])

$$
d(\phi)= \begin{cases}0 & \text { if } I_{F} \subset \operatorname{ker}(\phi), \\ \frac{a(\phi)}{n}-1 & \text { otherwise. }\end{cases}
$$

Let $\pi$ be an irreducible representation of $\mathrm{GL}_{m}(D)$. Let $\epsilon(s, \pi, \psi)$ denote the Godement-Jacquet local constant GoJa]. It takes the form

$$
\epsilon(s, \pi, \psi)=\epsilon(0, \pi, \psi) q^{-f(\pi, \psi) s}, \quad \text { where } \epsilon(0, \pi, \psi) \in \mathbb{C}^{\times} .
$$

A representation of $D^{\times}$is called unramified if it is trivial on $\mathfrak{o}_{D}^{\times}$. An unramified representation of $D^{\times}$is a character and has depth zero.

Let $\pi$ be a supercuspidal irreducible representation of $G$. We have (see ABPS3, Proposition 2.6]:

$$
f(\pi, \psi)= \begin{cases}n(c(\psi)+1)-1 & \text { if } m=1 \text { and } \pi \text { is unramified } \\ n(d(\pi)+1+c(\psi)) & \text { otherwise. }\end{cases}
$$

We set

$$
f(\pi):=f(\pi, \psi)-n c(\psi)
$$


Theorem 3.9. ABPS3, Theorem 2.7]

The depth $d(\pi)$ and the conductor $f(\pi)$ of each essentially square-integrable irreducible representation $\pi$ of $\mathrm{GL}_{m}(D)$ are linked by the following relation:

$$
d(\pi)= \begin{cases}0 & \text { if } \pi \text { is an unramified twist of } \operatorname{St}_{\mathrm{GL}_{m}(D)}, \\ \frac{f(\pi)-n}{n} & \text { otherwise. }\end{cases}
$$

In particular

$$
d(\pi)=\max \left\{\frac{f(\pi)-n}{n}, 0\right\} .
$$

Theorem 3.9 is a key ingredient in the proof of the following result:

Theorem 3.10. ABPS3, Theorem 2.9]

The LLC for $G=\mathrm{GL}_{m}(D)$ preserves the depth, that is:

$$
d(\pi)=d\left(\phi_{\pi}\right), \quad \text { where } \phi_{\pi}=\operatorname{rec}_{G}(\pi) .
$$

3.4.2. Inner forms of special general linear groups. The situation is different for $\mathrm{SL}_{m}(D)$. All the irreducible representations in a given $L$-packet $\Pi_{\phi}$ have the same depth, so the depth is an invariant of the $L$-packet, say $d\left(\Pi_{\phi}\right)$. We have $d\left(\Pi_{\phi}\right)=d(\varphi)$ where $\varphi$ is a lift of $\phi$ which has minimal depth among the lifts of $\phi$, and the following holds:

$$
d(\phi) \leq d\left(\Pi_{\phi}\right)
$$

for any Langlands parameter $\phi$ for $\mathrm{SL}_{m}(D)$ ABPS3, Proposition 3.4 and Corollary 3.4]. Moreover (34) is an equality if $\phi$ is essentially tame (in the terminology of Definition 2.13).

Remark 3.11. The notion of essentially tameness is consistent with the usual notion for Langlands parameters for $\mathrm{GL}_{n}(F)$. Indeed, any lift $\varphi: W_{F} \rightarrow \mathrm{GL}_{n}(\mathbb{C})$ of $\phi$, is called essentially tame if its restriction to $P_{F}$ is a direct sum of characters. We denote by $t(\varphi)$ the torsion number of $\varphi$, that is, the number of unramified characters $\chi$ of $W_{F}$ such $\varphi \chi \cong \varphi$. Then $\phi$ and $\varphi$ are essentially tame if and only if the residual characteristic $p$ of $F$ does not divide $n / t(\varphi)$ [BuHe, Appendix].

However, let $F$ be a local non-archimedean field of characteristic 2 , that is $F$ is of the form $F=\mathbb{F}_{q}((t))$, the field of Laurent series with coefficients in $\mathbb{F}_{q}$, with $q=2^{f}$. This case is particularly interesting because there are countably many quadratic extensions of $\mathbb{F}_{q}((t))$. Then equality holds in (34) only if $\phi$ is essentially tame (i.e., $t(\varphi)=2)$, as proved in AMPS.

In the case when $G$ is a classical group, the characteristic of $F$ is zero (that is, $F$ is a finite extension of $\mathbb{Q}_{p}$ ) and $p$ is odd, Ganapathy and Varma proved in GaVa, Lemma 8.2.3] that the following inequality holds

$$
d\left(\phi_{\pi}\right) \leq\lfloor d(\pi)\rfloor+1
$$

where $\phi$ the Langlands parameter attached to $\pi$ by Arthur. If moreover $p$ is sufficiently large with respect to $G$, then it is shown in GaVa that

$$
d(\pi) \leq d\left(\phi_{\pi}\right) .
$$

Very recently, it was proved in [Oi], for a quasi-split classical group over $F$, with $F$ of characteristic equal to zero and $p$ sufficiently large, that the depth of representations 
in each $L$-packet equals the depth of the corresponding $L$-parameter, and that, for quasi-split unitary groups, the depth is constant in each $L$-packet.

\section{Generalized Springer correspondence}

4.1. Cuspidal enhanced unipotent classes. Let $\mathcal{G}$ be a complex (possibly disconnected) reductive group. Let $\mathcal{G}^{\circ}$ be its identity component. For $u \in \mathcal{G}$ unipotent, we denote by $A_{\mathcal{G}}(u)$ the component group of the centralizer of $u$ in $\mathcal{G}$. We denote by $\mathrm{U}(\mathcal{G})$ the unipotent variety of $\mathcal{G}$.

Definition 4.1. The enhancement of $\mathrm{U}(\mathcal{G})$ is the set $\mathrm{U}_{\mathrm{e}}(\mathcal{G})$ of $\mathcal{G}$-conjugacy classes of pairs $(u, \rho)$, with $u \in \mathcal{G}$ unipotent and $\rho \in \operatorname{Irr}\left(A_{\mathcal{G}}(u)\right.$. We call a pair $(u, \rho)$ an enhanced unipotent class.

Let $D_{\mathcal{G}}^{b}(\mathrm{U}(\mathcal{G}))$ denote the constructible $\mathcal{G}$-equivariant derived category on $\mathrm{U}(\mathcal{G})$ defined by Bernstein and Lunts in $\left[\mathrm{BeLu}\right.$, and let $\operatorname{Perv}_{\mathcal{G}}(\mathrm{U}(\mathcal{G}))$ be its subcategory of $\mathcal{G}$ equivariant perverse sheaves.

By a $\mathcal{P}$-resolution of an algebraic variety $X$ we mean a variety $Y$ endowed with a free $\mathcal{P}$-action and a smooth $\mathcal{P}$-equivariant morphism $Y \rightarrow X$.

Definition 4.2. The integration functor is the functor

$$
\gamma_{\mathcal{P}}^{\mathcal{G}}: D_{\mathcal{P}}^{b}(\mathrm{U}(\mathcal{G})) \rightarrow D_{\mathcal{G}}^{b}(\mathrm{U}(\mathcal{G}))
$$

defined by

$$
\left(\gamma_{\mathcal{P}}^{\mathcal{G}} A\right)(Y):=\left(q_{Y}\right) ! A(Y)[2 \operatorname{dim} \mathcal{G} / \mathcal{P}]
$$

for $A$ any object of $D_{\mathcal{P}}^{b}(\mathrm{U}(\mathcal{G}))$ and $Y$ a $\mathcal{G}$-resolution of $\mathrm{U}(\mathcal{G})$, where $q_{Y}: \mathcal{P} \backslash Y \rightarrow \mathcal{G} \backslash Y$ is the quotient functor and $A(Y)$ is defined by regarding $Y$ as a $\mathcal{P}$-resolution of $\mathrm{U}(\mathcal{G})$.

Definition 4.3. Let $\mathcal{P}^{\circ}=\mathcal{L}^{\circ} \mathcal{U}$ be a parabolic subgroup of $\mathcal{G}^{\circ}$. Let

$$
m: \mathrm{U}\left(\mathcal{P}^{\circ}\right) \hookrightarrow \mathrm{U}\left(\mathcal{G}^{\circ}\right) \text { and } p: \mathrm{U}\left(\mathcal{P}^{\circ}\right) \rightarrow \mathrm{U}\left(\mathcal{L}^{\circ}\right)
$$

denote inclusion and projection, respectively. The parabolic induction functor is the functor

$$
\mathrm{i}_{\mathcal{L}^{\circ} \subset \mathcal{P}^{\circ}}^{\circ}:=\gamma_{\mathcal{P}^{\circ}}^{\mathcal{G}^{\circ}} \circ m_{!} \circ p^{*}: \operatorname{Perv}_{\mathcal{L}^{\circ}}\left(\mathrm{U}\left(\mathcal{L}^{\circ}\right)\right) \rightarrow \operatorname{Perv}_{\mathcal{G}^{\circ}}\left(\mathrm{U}\left(\mathcal{G}^{\circ}\right)\right) .
$$

\section{Remarks 4.4.}

(1) The functor $\mathrm{i}_{\mathcal{L}^{\circ} \subset \mathcal{P}^{\circ}}^{\circ}$ commutes with Verdier duality. It is left adjoint to $\mathrm{r}_{\mathcal{L}^{\circ} \subset \mathcal{P}^{\circ}}^{\mathcal{G}^{\circ}}:=p_{!} \circ m^{*}$ and right adjoint to ${ }^{\prime} \mathrm{r}_{\mathcal{L}^{\circ} \subset \mathcal{P}^{\circ}}^{\mathcal{G}^{\circ}}:=p_{*} \circ m^{!}$. These functors are exchanged by Verdier duality.

(2) If $\mathcal{F}_{L}$ is a simple object in $\operatorname{Perv}_{\mathcal{L}^{\circ}}\left(\mathrm{U}\left(\mathcal{L}^{\circ}\right)\right)$, then $\mathrm{i}_{\mathcal{L}^{\circ} \subset \mathcal{P}^{\circ}}\left(\mathcal{F}_{\mathcal{L}^{\circ}}\right)$ is semisimple.

Definition 4.5. A simple object $\mathcal{F}$ in $\operatorname{Perv}_{\mathcal{G}}{ }^{\circ}\left(\mathrm{U}\left(\mathcal{G}^{\circ}\right)\right)$ is cuspidal if for any simple object $\mathcal{F}_{\mathcal{L}^{\circ}}$ in $\operatorname{Perv} \mathcal{L}^{\circ}\left(\mathrm{U}\left(\mathcal{L}^{\circ}\right)\right), \mathcal{F}$ does not occur in $\mathrm{i}_{\mathcal{L}^{\circ} \subset \mathcal{P}^{\circ}}\left(\mathcal{F}_{\mathcal{L}^{\circ}}\right)$ (equivalently, if $\mathrm{r}_{\mathcal{L}^{\circ} \subset \mathcal{P}^{\circ}}^{\mathcal{G}^{\circ}}(\mathcal{F})=0$, resp, $\left.{ }^{\prime}{ }_{\mathcal{L}^{\circ} \subset \mathcal{P}^{\circ}}^{\circ}(\mathcal{F})=0\right)$ for any proper parabolic subgroup $\mathcal{P}^{\circ}$ of $\mathcal{G}^{\circ}$, with Levi factor $\mathcal{L}^{\circ}$.

\section{Remarks 4.6.}

(1) Cuspidality is preserved by Verdier duality (see for instance [AHJR, Remark 2.3]).

(2) The above definition of cuspidality is inspired by [Lus3] (see also [AHJR]). It is equivalent to the original definition of cuspidality given by Lusztig in 1984 in [Lus2] (as shown in [Lus6, 23.2. (b)]). 
For $u$ a given unipotent element in $\mathcal{G}^{\circ}$, let $A_{\mathcal{G}}(u)$ denote the component group of its centralizer $\mathrm{Z}_{\mathcal{G}^{\circ}}(u)$ of $u$ in $\mathcal{G}^{\circ}$. Let $\mathcal{O}_{u}=(u)_{\mathcal{G}^{\circ}}$ be the $\mathcal{G}^{\circ}$-conjugacy class of $u$. We set $A_{\mathcal{G}^{\circ}}\left(\mathcal{O}_{u}\right):=A_{\mathcal{G}^{\circ}}(u)$. We will denote by

$$
\rho \mapsto \mathcal{E}_{\rho}
$$

the bijection between $\operatorname{Irr}\left(A_{\mathcal{G}} \circ(u)\right)$ and the irreducible $\mathcal{G}^{\circ}$-equivariant local systems $\mathcal{E}$ on $\mathcal{O}_{u}$. We denote by

$$
\mathcal{E} \mapsto \rho_{\mathcal{E}}
$$

the inverse bijection.

\section{Definition 4.7.}

(1) A character $\rho \in \operatorname{Irr}\left(A_{\mathcal{G}^{\circ}}(u)\right)$ is cuspidal if the perverse $\operatorname{sheaf} \operatorname{IC}\left(\mathcal{O}, \mathcal{E}_{\rho}\right)$ is cuspidal.

(2) An enhanced unipotent class $(\mathcal{O}, \rho)$ in $\mathcal{G}^{\circ}$ is cuspidal if $\rho$ is cuspidal.

Proposition 4.8. Lus2, Proposition 2.8] If $\left(\mathcal{O}_{u}, \rho\right)$ is cuspidal, then $u$ is a distinguished unipotent element in $\mathcal{G}^{\circ}$ (i.e., $u$ does not meet $\mathrm{U}\left(\mathcal{L}^{\circ}\right)$ for any proper Levi subgroup $\mathcal{L}$ of $\left.\mathcal{G}^{\circ}\right)$.

Remark 4.9. In general not every distinguished unipotent element supports a cuspidal representation.

Example 4.10. For $\mathcal{G}=\mathrm{SL}_{n}(\mathbb{C})$, the unipotent classes in $\mathcal{G}$ are in bijection with the partitions $\lambda=\left(\lambda_{1}, \lambda_{2}, \ldots, \lambda_{r}\right)$ of $n$ : the corresponding $\mathcal{G}$-conjugacy class $\mathcal{O}_{\lambda}$ consists of unipotent matrices with Jordan blocks of sizes $\lambda_{1}, \lambda_{2}, \ldots, \lambda_{r}$. We identify the center $\mathrm{Z}(\mathcal{G})$ with the group $\mu_{n}$ of complex $n$-roots of unity. For $u \in \mathcal{O}_{\lambda}$, the natural homomorphism $\mathrm{Z}(\mathcal{G}) \rightarrow A_{\mathcal{G}}(u)$ is surjective with kernel $\mu_{n / \operatorname{gcd}(\lambda)}$, where $\operatorname{gcd}(\lambda):=\operatorname{gcd}\left(\lambda_{1}, \lambda_{2}, \ldots, \lambda_{r}\right)$. Hence the irreducible $\mathcal{G}$-equivariant local systems on $\mathcal{O}_{\lambda}$ all have rank one, and they are distinguished by their central characters, which range over those $\chi \in \operatorname{Irr}\left(\mu_{n}\right)$ such $\operatorname{gcd}(\lambda)$ is a multiple of the order of $\chi$. We denote these local systems by $\mathcal{E}_{\lambda, \chi}$. The unique distinguished unipotent class in $\mathcal{G}$ is the regular unipotent class $\mathcal{O}_{(n)}$, consisting of unipotent matrices with a single Jordan block. The cuspidal irreducible $G$-equivariant local systems are supported on $\mathcal{O}_{(n)}$ (by Proposition 4.8) and are of the form $\mathcal{E}_{(n), \chi}$, with $\chi \in \operatorname{Irr}\left(\mu_{n}\right)$ of order $n$ (see [Lus2, (10.3.2)]).

We will now extend the above notion of cuspidality from $\mathcal{G}^{\circ}$ to $\mathcal{G}$ :

Definition 4.11. An enhanced unipotent class $(\mathcal{O}, \rho)$ with $\mathcal{O}=(u)_{\mathcal{G}}$ and $\rho \in$ $\operatorname{Irr}\left(A_{\mathcal{G}}(u)\right)$ is cuspidal if the restriction of $\rho$ to $A_{\mathcal{G}} \circ(u)$ is a direct sum of cuspidal irreducible representations of $A_{\mathcal{G}^{\circ}}(u)$.

Notation 4.12. We set

$$
\operatorname{Irr}_{\text {cusp }}\left(A_{\mathcal{G}}(u)\right):=\left\{\epsilon \in \operatorname{Irr}\left(A_{\mathcal{G}}(u)\right) \text { such that } \epsilon \text { is cuspidal }\right\} .
$$

Definition 4.13. A quasi Levi subgroup of $\mathcal{G}$ is a subgroup $\mathcal{M}$ of the form $\mathcal{M}=$ $\mathrm{Z}_{\mathcal{G}}\left(\mathrm{Z}(\mathcal{L})^{\circ}\right)$, with $\mathcal{L}$ a Levi subgroup of $\mathcal{G}^{\circ}$. The group $\mathcal{M}$ is said to be cuspidal if there exists a cuspidal enhanced unipotent pair in $\mathcal{M}$.

Remark 4.14. A Levi subgroup of $\mathcal{G}$ is a quasi-Levi subgroup, and in the case when $\mathcal{G}$ connected, both notions coincide. 
Notation 4.15. Let $\mathfrak{B}\left(\mathrm{U}_{\mathrm{e}}(\mathcal{G})\right)$ be the set of $\mathcal{G}$-conjugacy classes of pairs $(\mathcal{M},(\mathcal{O}, \epsilon))$, where $\mathcal{M}$ is a cuspidal quasi-Levi subgroup of $\mathcal{G}$, and $(\mathcal{O}, \epsilon)$ is a cuspidal enhanced unipotent pair in $\mathcal{M}$.

4.2. A partition of the enhancement of the unipotent variety of $\mathcal{G}^{\circ}$. The purpose of this section is to describe a theory à la Harish-Chandra for $\mathrm{U}_{\mathrm{e}}\left(\mathcal{G}^{\circ}\right)$, the first step being to define a cuspidal support map for enhanced unipotent classes of $\mathcal{G}$.

The enhancement $\mathrm{U}_{\mathrm{e}}\left(\mathcal{G}^{\circ}\right)$ of $\mathrm{U}\left(\mathcal{G}^{\circ}\right)$ parametrizes the isomorphism classes of simple objects of $\operatorname{Perv}_{\mathcal{G}^{\circ}}\left(\mathrm{U}\left(\mathcal{G}^{\circ}\right)\right)$. Indeed, the simple objects in $\operatorname{Perv}_{\mathcal{G}^{\circ}}\left(\mathrm{U}\left(\mathcal{G}^{\circ}\right)\right)$ are the $\mathrm{IC}(\mathcal{O}, \mathcal{E})$, where $\mathcal{O}$ is a unipotent class in $\mathcal{G}^{\circ}$ and $\mathcal{E}$ is an irreducible $\mathcal{G}^{\circ}$-equivariant $\overline{\mathbb{Q}}_{\ell}$-local system on $\mathcal{O}$.

Let $(\mathcal{O}, \rho) \in \mathrm{U}_{\mathrm{e}}\left(\mathcal{G}^{\circ}\right)$ be an arbitrary enhanced unipotent class, and set $\mathcal{F}_{\rho}:=$ $\operatorname{IC}\left(\mathcal{O}, \mathcal{E}_{\rho}\right)$. Then $\mathcal{F}_{\rho}$ occurs as a summand of $\mathrm{i}_{\mathcal{L}^{\circ} \subset \mathcal{P}^{\circ}}\left(\operatorname{IC}\left(\mathcal{O}_{0}, \mathcal{E}_{0}\right)\right)$, for some quadruple $\left(\mathcal{P}^{\circ}, \mathcal{L}^{\circ}, \mathcal{O}_{0}, \mathcal{E}_{0}\right)$, where $\mathcal{P}^{\circ}$ is a parabolic subgroup of $\mathcal{G}^{\circ}$ with Levi subgroup $\mathcal{L}^{\circ}$ and $\left(\mathcal{O}_{0}, \mathcal{E}_{0}\right)$ is a cuspidal enhanced unipotent class in $\mathcal{L}^{\circ}$ (see [Lus2, $\S 6.2$ ] and AHJR, Cor. 2.7]) and, moreover, $\left(\mathcal{P}^{\circ}, \mathcal{L}^{\circ}, \mathcal{O}_{0}, \mathcal{E}_{0}\right)$ is unique up to $\mathcal{G}^{\circ}$-conjugation (see [Lus2, Prop. 6.3]). We set $\epsilon:=\rho_{\mathcal{E}_{0}}$ using the bijection (38), and we denote by $\mathfrak{t}^{\circ}:=\left(\mathcal{L}^{\circ},\left(\mathcal{O}_{0}, \epsilon\right)\right)_{\mathcal{G}^{\circ}}$, the $\mathcal{G}^{\circ}$-conjugacy class of $\left(\mathcal{L}^{\circ},\left(\mathcal{O}_{0}, \epsilon\right)\right)$ and we call it the cuspidal support of the enhanced unipotent class $(\mathcal{O}, \rho)$.

The center $\mathrm{Z}\left(\mathcal{G}^{\circ}\right)$ of $\mathcal{G}^{\circ}$ maps naturally to $A_{\mathcal{G}^{\circ}}(\mathcal{O})$ and to $A_{\mathcal{L}^{\circ}}\left(\mathcal{O}_{0}\right)$. By construction [Lus2, Theorem 6.5.a]

$$
\rho \text { and } \epsilon \text { have the same } \mathrm{Z}\left(\mathcal{G}^{\circ}\right) \text {-character. }
$$

Definition 4.16. The cuspidal support map for $\mathrm{U}_{\mathrm{e}}\left(\mathcal{G}^{\circ}\right)$ is the map

$$
\Psi_{\mathcal{G}^{\circ}}: \mathrm{U}_{\mathrm{e}}\left(\mathcal{G}^{\circ}\right) \rightarrow \mathfrak{B}\left(\mathrm{U}_{\mathrm{e}}\left(\mathcal{G}^{\circ}\right)\right)
$$

where $\mathfrak{B}\left(\mathrm{U}_{\mathrm{e}}\left(\mathcal{G}^{\circ}\right)\right)$ is as in Notation 4.15 , which sends the $\mathcal{G}^{\circ}$-conjugacy class of $(\mathcal{O}, \rho)$ to its cuspidal support $\mathfrak{t}^{\circ}=\left(\mathcal{L}^{\circ},\left(\mathcal{O}_{0}, \epsilon\right)\right)_{\mathcal{G}^{\circ}}$.

By (39) the map $\Psi_{\mathcal{G}^{\circ}}$ preserves the $\mathrm{Z}\left(\mathcal{G}^{\circ}\right)$-characters of the involved representations.

Notation 4.17. Let $\mathfrak{t}^{\circ} \in \mathfrak{B}\left(\mathrm{U}_{\mathrm{e}}\left(\mathcal{G}^{\circ}\right)\right)$.

(1) We denote by $\mathrm{U}_{\mathrm{e}}\left(\mathcal{G}^{\circ}\right)^{\mathfrak{t}^{\circ}}$ denotes the fiber of $\mathfrak{t}$ under the map $\Psi_{\mathcal{G}^{\circ}}$.

(2) Let $W_{\mathcal{L}^{\circ}}:=\mathrm{N}_{\mathcal{G}^{\circ}}\left(\mathcal{L}^{\circ}\right) / \mathcal{L}^{\circ}$, and let $W_{\mathfrak{t}^{\circ}}:=\mathrm{N}_{\mathcal{G}^{\circ}}\left(\mathfrak{t}^{\circ}\right) / \mathcal{L}^{\circ}$.

Theorem 4.18. Lus2]

(1) The group $W_{\mathcal{L}^{\circ}}$ is a Weyl group and it coincides with $W_{\mathfrak{t}^{\circ}}$ for every $\mathfrak{t}^{\circ} \in$ $\mathfrak{B}\left(\mathrm{U}_{\mathrm{e}}\left(\mathcal{G}^{\circ}\right)\right)$.

(2) We have

$$
\mathrm{U}_{\mathrm{e}}\left(\mathcal{G}^{\circ}\right)=\bigsqcup_{\mathfrak{t}^{\circ} \in \mathfrak{B}\left(\mathrm{U}_{\mathrm{e}}\left(\mathcal{G}^{\circ}\right)\right)} \mathrm{U}_{\mathrm{e}}\left(\mathcal{G}^{\circ}\right)^{\mathfrak{t}^{\circ}}
$$

and $\mathrm{U}_{\mathrm{e}}\left(\mathcal{G}^{\circ}\right)^{\mathfrak{t}^{\circ}}$ is in bijection with $\operatorname{Irr}\left(W_{\mathcal{L}^{\circ}}\right)$.

The goal of the next two subsections is to generalize the previous notation and terminology from $\mathcal{G}^{\circ}$ to $\mathcal{G}$. 
4.3. Twisted group algebras. Throughout this section $\Gamma$ is a finite group and $K$ is an algebraically closed field whose characteristic does not divide the order of $\Gamma$. Suppose that $\kappa: \Gamma \times \Gamma \rightarrow K^{\times}$is a 2-cocycle, that is,

$$
\kappa\left(\gamma_{1}, \gamma_{2} \gamma_{3}\right) \kappa\left(\gamma_{2}, \gamma_{3}\right)=\kappa\left(\gamma_{1}, \gamma_{2}\right) \kappa\left(\gamma_{1} \gamma_{2}, \gamma_{3}\right) \quad \forall \gamma_{1}, \gamma_{2}, \gamma_{3} \in \Gamma .
$$

The $\kappa$-twisted group algebra of $\Gamma$ is defined to be the $K$-vector space $K[\Gamma, \kappa]$ with basis $\left\{T_{\gamma}: \gamma \in \Gamma\right\}$ and multiplication rules

$$
T_{\gamma} T_{\gamma^{\prime}}=\kappa\left(\gamma, \gamma^{\prime}\right) T_{\gamma \gamma^{\prime}} \quad \gamma, \gamma^{\prime} \in \Gamma .
$$

Its representations can be considered as projective $\Gamma$-representations. Schur showed (see [CuRe, Theorem 53.7]) that there exists a finite central extension $\tilde{\Gamma}$ of $\Gamma$, such that

- $\operatorname{char}(K)$ does not divide $|\tilde{\Gamma}|$,

- every irreducible projective $\Gamma$-representation over $K$ lifts to an irreducible $K$-linear representation of $\tilde{\Gamma}$.

Then $K[\Gamma, \kappa]$ is a direct summand of $K[\tilde{\Gamma}]$, namely the image of a minimal idempotent in $K[\operatorname{ker}(\tilde{\Gamma} \rightarrow \Gamma)]$. The condition on $\operatorname{char}(K)$ ensures that $K[\tilde{\Gamma}]$ is semisimple, so $K[\Gamma, \kappa]$ is also semisimple.

\subsection{A partition of the enhancement of the unipotent variety of $\mathcal{G}$.}

Notation 4.19. Let $\mathfrak{t}=\left(\mathcal{M},\left(\mathcal{O}_{0}, \epsilon\right)\right)_{\mathcal{G}} \in \mathfrak{B}\left(\mathrm{U}_{\mathrm{e}}(\mathcal{G})\right)$. We set

$$
W_{\mathfrak{t}}:=\mathrm{N}_{\mathcal{G}}(\tau) / \mathcal{M} \text { and } W_{\mathfrak{t}}^{\circ}:=\mathrm{N}_{\mathcal{G}^{\circ}}\left(\mathcal{M}^{\circ}\right) / \mathcal{M}^{\circ} \text {. }
$$

Theorem 4.20. [AMS1, §4] Let $(\mathcal{O}, \rho) \in \mathrm{U}_{\mathrm{e}}(\mathcal{G})$. There exists a 2-cocycle

$$
\kappa_{\mathfrak{t}}: W_{\mathfrak{t}} / W_{\mathfrak{t}}^{\circ} \times W_{\mathfrak{t}} / W_{\mathfrak{t}}^{\circ} \rightarrow \mathbb{C}^{\times}
$$

and a map, called the cuspidal support map for $\mathrm{U}_{\mathrm{e}}(\mathcal{G})$

$$
\Psi_{\mathcal{G}}: \mathrm{U}_{\mathrm{e}}(\mathcal{G}) \rightarrow \mathfrak{B}\left(\mathrm{U}_{\mathrm{e}}(\mathcal{G})\right)
$$

(which coincides to the map $\Psi_{\mathcal{G}}$ defined above in the case when $\mathcal{G}$ is connected), such that

$$
\mathrm{U}_{\mathrm{e}}(\mathcal{G})=\bigsqcup_{\mathfrak{t} \in \mathfrak{B}\left(\mathrm{U}_{\mathrm{e}}(\mathcal{G})\right)} \mathrm{U}_{\mathrm{e}}(\mathcal{G})^{\mathfrak{t}},
$$

in which the fiber $\mathrm{U}_{\mathrm{e}}(\mathcal{G})^{\mathfrak{t}}$ of $\mathfrak{t}$ under the map $\Psi_{\mathcal{G}}$ is isomorphic to the $\kappa_{\mathrm{t}}$-twisted version $\mathbb{C}\left[W_{\mathfrak{t}}, \kappa_{\mathfrak{t}}\right]$ of the group algebra $\mathbb{C}\left(W_{\mathfrak{t}}\right]$ of the finite group $W_{\mathfrak{t}}$.

Notation 4.21. Let $\Sigma_{\mathfrak{t}}$ denote the bijection

$$
\mathrm{U}_{\mathrm{e}}(\mathcal{G})^{\mathfrak{t}} \longrightarrow \mathbb{C}\left[W_{\mathfrak{t}}, \kappa_{\mathfrak{t}}\right]
$$

mentioned in Theorem 4.20,

Remark 4.22. When $\mathcal{G}$ is connected, the cocycle $\kappa_{\mathrm{t}}$ is trivial. When $\mathcal{G}$ is disconnected, the cocycle $\kappa_{\mathrm{t}}$ is not always trivial.

An intuitive way of thinking of Theorem 4.20 is to view the set $\mathfrak{B}\left(\mathrm{U}_{\mathrm{e}}(\mathcal{G})\right)$ as a "palette of colors" and the map $\Psi_{\mathcal{G}}$ as a way to paint the elements of the fiber $\mathrm{U}_{\mathrm{e}}(\mathcal{G})^{\mathfrak{t}}$ of $\mathfrak{t}$ under $\Psi_{\mathcal{G}}$ in the same color as $\mathfrak{t}$. Moreover, for each color, the subset of elements of $\mathrm{U}_{\mathrm{e}}(\mathcal{G})$ with that color has a nice structure (here, that of the twisted group algebra). 


\section{Cuspidality for enhanced $L$-Parameters: Definition And COnjeCture}

Definition 5.1. An enhanced $L$-parameter $(\phi, \rho) \in \Phi(G)_{\mathrm{e}}$ is called cuspidal if $\phi$ is discrete and $\left(u_{\phi}, \rho\right)$ is a cuspidal enhanced unipotent class in $\mathcal{G}_{\lambda_{\phi}}$ in the terminology of Definition 4.11.

Recall that an irreducible smooth complex representation of the group $G$ is called supercuspidal if it does not appear in any $G$-representation induced from a proper Levi subgroup of $G$. Bernstein [BeDe, $\S 2$ ] realized that an irreducible $G$ representation is supercuspidal if and only if it is compact. Here compact means that the representation behaves like one of a compact group, in the sense that all its matrix coefficients have compact support modulo the centre of $G$.

Conjecture of cuspidality 5.2. [AMS1, $\S 6]$

The cuspidal enhanced Langlands parameters correspond by the LLC to the irreducible supercuspidal representations of $G$.

Conjecture 5.2 is known to be true in the following cases:

- for general linear groups and split classical groups (any representation), with $F$ of characteristic equal to 0: Mou1,

- for inner forms of linear groups and of special linear groups, quasi-split unitary groups (any representation) with $F$ of characteristic equal to 0 , and for Deligne-Lusztig depth-zero representations: AMS1, § 6],

- for the representations with unipotent reduction of the group $G$ of the $F$ rational points of a connected reductive algebraic group which splits over an unramified extension of $F$ : [FOS, Theorem 2] (when $G$ is simple of adjoint type it is a special case of [Lus4], [Lus5]).

\section{A partition of the set of enhanced Langlands parameters}

We shall define a similar partition of the set of enhanced Langlands parameters by plugging the above construction into the framework of the Langlands correspondence. Let $(\phi, \rho) \in \Phi\left({ }^{L} G\right)$. We take for $\mathcal{G}$ the group $\mathcal{G}_{\phi}$ defined in (18) and set

$$
\left[\mathcal{M}_{\phi},\left(\mathcal{O}_{0}, \epsilon\right)\right]_{\mathcal{G}_{\phi}}:=\Psi_{\mathcal{G}_{\phi}}\left(u_{\phi}, \rho\right) \in \mathfrak{B}\left(\mathrm{U}_{\mathrm{e}}\left(\mathcal{G}_{\phi}\right)\right)
$$

where the map $\Psi_{\mathcal{G}_{\phi}}$ is that of Theorem 4.20. Hence $\mathcal{M}_{\phi}$ is a quasi Levi subgroup of $\mathcal{G}_{\phi}$ and $\left(\mathcal{O}_{0}, \epsilon\right)$ is a cuspidal enhanced unipotent class in $\mathcal{M}_{\phi}$. For simplicity, we will often refer to the unipotent class $\mathcal{O}_{0}$ in $\mathcal{M}_{\phi}$ by a unipotent element $v$ in it.

The idea is very similar as above: using the data defined in $(43)$, we will construct a set $\mathfrak{B}^{\vee}(G)$ which will play the role of the palette of colors for $\Phi_{\mathrm{e}}\left({ }^{L} G\right)$, and we will define a "way to paint" the elements in $\Phi_{\mathrm{e}}\left({ }^{L} G\right)$ with this set of colors (that is, we will construct a cuspidal support map) such there is a decomposition

$$
\Phi_{\mathrm{e}}\left({ }^{L} G\right)=\bigsqcup_{\mathfrak{s}^{\vee} \in \mathfrak{B}^{\vee}(\mathbf{G})} \Phi_{\mathrm{e}}\left({ }^{L} G\right)^{\mathfrak{s}^{\vee}}
$$

where, for each $\mathfrak{s}^{\vee}$ in $\mathfrak{B}^{\vee}(\mathbf{G})$, the subset $\Phi_{\mathrm{e}}\left({ }^{L} G\right)^{\mathfrak{s}^{\vee}}$ of elements with color $\mathfrak{s}^{\vee}$ is related to a finite collection ${ }^{1}$ of generalized affine Hecke algebras with possibly unequal parameters.

\footnotetext{
${ }^{1}$ This reflects the fact that we consider simultaneously all the inner twists of a given group $G$.
} 
Definition 6.1. Let $\mathbf{T}^{\vee} \subset \mathbf{G}^{\vee}$ be a torus such that the projection $\mathrm{Z}_{L_{G}}(T) \rightarrow W_{F}$ is surjective. Then we call $\mathrm{Z}_{L_{G}}(T)$ a Levi $L$-subgroup of ${ }^{L} G$.

Remark 6.2. In Bor] such groups are called Levi subgroups of ${ }^{L} G$, however we prefer to stick to the connectedness of Levi subgroups.

Remark 6.3. Choose a $W_{F}$-stable pinning for $\mathbf{G}^{\vee}$. This defines the notion of standard Levi subgroups of $\mathbf{G}^{\vee}$. An alternative characterization of the Levi $L$ subgroups of ${ }^{L} G$ is as follows [AMS1, Lem]: Let $\mathrm{Z}_{L_{G}}(T)$ be a Levi $L$-subgroup of ${ }^{L} G$. There exists a $W_{F^{-}}$-stable standard Levi subgroup $\mathbf{L}^{\vee}$ of $\mathbf{G}^{\vee}$ such that $Z_{L_{G}}(T)$ is $\mathbf{G}^{\vee}$-conjugate to $\mathbf{L}^{\vee} \rtimes W_{F}=:{ }^{L} L$ and $Z_{L_{G}}(T) \cap \mathbf{G}^{\vee}$ is conjugate to $\mathbf{L}^{\vee}$.

Conversely, every $\mathbf{G}^{\vee}$-conjugate of this ${ }^{L} L$ is a Levi $L$-subgroup of ${ }^{L} G$.

Notation 6.4. In the sequel, we will use the (slightly abusive) notation ${ }^{L} L$ for an arbitrary $L$-Levi of $G$.

Definition 6.5. Let ${ }^{L} L$ be a Levi $L$-subgroup of ${ }^{L} G$.

(1) A Langlands parameter for ${ }^{L} L$ is a group homomorphism $\phi: W_{F}^{\prime} \rightarrow{ }^{L} L$ satisfying the requirements of Definition 2.12 .

(2) An enhancement of $\phi$ is an irreducible representation $\rho$ of $\pi_{0}\left(\mathrm{Z}_{\mathbf{L}^{\vee}}^{1}(\phi)\right)$, where $\mathbf{L}_{\mathrm{sc}}^{\vee}$ is the simply connected cover of the derived group of $\mathbf{L}^{\vee}={ }^{L} \mathbf{L}^{\vee} \cap \mathbf{G}^{\vee}$.

The group $\mathbf{L}^{\vee}$ acts on the collection of enhanced $L$-parameters for ${ }^{L} L$ by 19 .

Definition 6.6. We say that an enhanced $L$-parameter $(\phi, \rho)$ for ${ }^{L} L$ is cuspidal if $\phi$ is discrete for ${ }^{L} L$ and $\left(u_{\phi}=\phi\left(1,\left(\begin{array}{ll}1 & 1 \\ 0 & 1\end{array}\right)\right), \rho\right)$ is a cuspidal pair for $Z_{\mathbf{L}_{\mathrm{sc}}^{\vee}}^{1}\left(\left.\phi\right|_{W_{F}}\right)$.

Notation 6.7. We denote the set of $\mathbf{L}^{\vee}$-orbits by $\Phi_{\mathrm{e}}\left({ }^{L} L\right)$ and the subset of cuspidal $\mathbf{L}^{\vee}$-orbits by $\Phi_{\text {e,cusp }}\left({ }^{L} L\right)$.

Let $\mathbf{L}^{\vee}$ be a Levi subgroup of $\mathbf{G}^{\vee}$, and let $\mathbf{L}_{\mathrm{c}}^{\vee}$ denote the pre-image of $L^{\vee}$ under under $\mathbf{G}_{\mathrm{sc}}^{\vee} \rightarrow \mathbf{G}^{\vee}$. The derived group of $\mathbf{L}_{\mathrm{c}}^{\vee}$ is the simply connected cover of $\mathbf{L}_{\mathrm{der}}^{\vee}$, and we identify $\mathbf{L}_{\mathrm{sc}}^{\vee}$ with the inverse image of $\mathbf{L}_{\text {der }}^{\vee}$ under $\mathbf{G}_{\mathrm{sc}}^{\vee} \rightarrow \mathbf{G}^{\vee}$.

Definition 6.8. A cuspidal datum for ${ }^{L} G$ is a triple $\left({ }^{L} L, \phi, \rho\right)$ where ${ }^{L} L$ is a Levi $L$-subgroup of ${ }^{L} G$, such that $(\phi, \rho)$ is cuspidal for ${ }^{L} L$. It is relevant for $G$ if

- $\rho=\zeta$ on $\mathbf{L}_{\mathrm{sc}}^{\vee} \cap \mathrm{Z}\left(\mathbf{G}_{\mathrm{sc}}^{\vee}\right)^{W_{F}}$, where $\zeta \in \operatorname{Irr}\left(\mathrm{Z}\left(\mathbf{G}_{\mathrm{sc}}^{\vee}\right)^{W_{F}}\right)$ parametrizes the inner twist $G$ of $G^{*}$ via the Kottwitz isomorphism (9):

- $\rho=1$ on $\mathbf{L}_{\mathrm{sc}}^{\vee} \cap \mathrm{Z}\left(\mathbf{L}_{\mathrm{c}}^{\vee}\right)^{\circ}$.

We will give now the technical details of the construction. Upon replacing $(\phi, \rho)$ by a $\mathbf{G}^{\vee}$-conjugate, there exists a Levi subgroup $L$ of $G$ such that $\left(\phi_{\mid W_{F}}, v, \epsilon\right)$ is a cuspidal enhanced Langlands parameter for $L$, and

$$
{ }^{L} L:=\mathbf{L}^{\vee} \rtimes W_{F}=\mathrm{Z}_{L_{G}}\left(\mathrm{Z}_{\mathcal{M}_{\phi}}^{\circ}\right) .
$$

Then we set

$$
{ }^{L} \Psi(\phi, \rho):=\left[{ }^{L} L,\left(\phi_{\mid W_{F}}, v, \epsilon\right)\right]_{\mathbf{G}^{\vee}} .
$$

The group of unramified characters of $L$ is naturally isomorphic to $\left(\mathrm{Z}_{\mathbf{L} \vee \rtimes I_{F}}\right)_{W_{F}}^{\circ}$. Denote the latter by $X_{\mathrm{nr}}\left({ }^{L} L\right)$. Given $(\varphi, \epsilon) \in \Phi_{\mathrm{e}}\left(\mathbf{L}^{\vee}, F\right)$, and $\xi \in X_{\mathrm{nr}}\left({ }^{L} L\right)$, define $(\xi \varphi, \varrho) \in \Phi_{\mathrm{e}}\left(\mathbf{L}^{\vee}, F\right)$ by $\xi \varphi:=\varphi$ on $I_{F} \times \mathrm{SL}_{2}(\mathbb{C})$ and $(\xi \varphi)\left(\operatorname{Fr}_{F}\right):=\tilde{\xi} \varphi\left(\operatorname{Fr}_{F}\right)$, where $\tilde{\xi} \in \mathrm{Z}_{L^{\vee} \rtimes I_{F}}^{\circ}$ represents $z$. 
Definition 6.9. We denote by $\mathfrak{s}^{\vee}$ the $\mathbf{G}^{\vee}$-conjugacy class of $\left({ }^{L} L, X_{\mathrm{nr}}\left({ }^{L} L\right) \cdot(\varphi, \epsilon)\right)$, where $L$ is a Levi subgroup of $G$, and $(\varphi, \epsilon)$ is a cuspidal enhanced Langlands parameter for $L$. We write

$$
\mathfrak{s}^{\vee}=\mathfrak{s}_{G}^{\vee}=\left[{ }^{L} L,(\varphi, \epsilon)\right]_{G^{\vee}} .
$$

We call $\mathfrak{s}^{\vee}$ an inertial class for $\Phi_{\mathrm{e}}\left(\mathbf{G}^{\vee}, F\right)$ and denote by $\mathfrak{B}^{\vee}(\mathbf{G})$ the set of such $\mathfrak{s}^{\vee}$.

Then the subset

$$
\Phi_{\mathrm{e}}\left({ }^{L} G\right)^{\mathfrak{s}^{\vee}}:=\left({ }^{L} \Psi\right)^{-1}\left(\mathbf{L}^{\vee} \rtimes W_{F}, \mathfrak{s}_{L}^{\vee}\right)
$$

of $\Phi_{\mathrm{e}}\left({ }^{L} G\right)$ is in bijection with the simple modules of a finite collection of twisted extended affine Hecke algebra $\mathcal{H}\left(\mathfrak{s}^{\vee}, \overrightarrow{\mathbf{z}}\right)_{\vartheta}$ with $\vartheta \in H^{1}\left(F, \mathbf{G}_{\text {ad }}^{*}\right)$ : it is the object of AMS2] and [AMS3].

6.1. A generalized Springer correspondence for enhanced $L$-parameters. Let $(\phi, \rho)$ be an enhanced $L$-parameter for $G$ and write as above $\Psi_{\mathcal{G}_{\phi}}\left(u_{\phi}, \rho\right)=$ : $\left[\mathcal{M}_{\phi}, v, \epsilon\right]_{\mathcal{G}_{\phi}}$. Up to $\mathcal{G}_{\phi}$-conjugacy there exists a unique $\gamma_{v}: \mathrm{SL}_{2}(\mathbb{C}) \rightarrow \mathcal{M}_{\phi}^{\circ}$ adapted to $\phi$. Moreover the cocharacter

$$
\chi_{\phi, v}: z \mapsto \phi\left(1,\left(\begin{array}{cc}
z & 0 \\
0 & z^{-1}
\end{array}\right)\right) \gamma_{v}\left(\begin{array}{cc}
z^{-1} & 0 \\
0 & z
\end{array}\right)
$$

has image in $\mathrm{Z}\left(\mathcal{M}_{\phi}\right)^{\circ}$.

Fix a $G$-relevant cuspidal datum $\left({ }^{L} L, \phi_{v}, \epsilon\right)$ for ${ }^{L} G$, and write

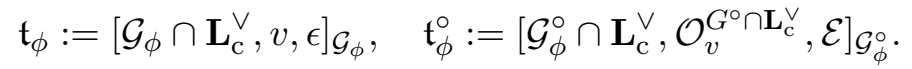

The next result may be viewed as a version of the generalized Springer correspondence for enhanced $L$-parameters instead of enhaced unipotent classes.

Proposition 6.10. AMS1, Proposition 9.1]

(a) There is a bijection

$$
\begin{array}{cccc}
{ }^{L} \Sigma_{\mathfrak{t}_{\phi}}: & { }^{L} \Psi^{-1}\left({ }^{L} L, \phi_{v}, q \epsilon\right) & \longleftrightarrow & \operatorname{Irr}\left(\mathbb{C}\left[W_{\mathfrak{t}_{\phi}}, \kappa_{\mathfrak{t}_{\phi}}\right]\right) \\
(\phi, \rho) & \mapsto & \Sigma_{\mathfrak{t}_{\phi}}\left(u_{\phi}, \rho\right) \\
\left(\left.\phi\right|_{\mathbf{W}_{F}}, \Sigma_{\mathfrak{t}_{\phi}}^{-1}(E)\right) & \longleftrightarrow & E,
\end{array}
$$

where $\Sigma_{\mathfrak{t}_{\phi}}$ is the bijection defined in Notation 4.21.

(b) The canonical bijection $\Sigma_{\mathfrak{t}_{\phi}^{\circ}}$ between $\Psi_{\mathcal{G}^{\circ}}^{-1}\left(\mathfrak{t}_{\phi}^{\circ}\right) \subset \mathrm{U}_{\mathrm{e}}\left(\mathcal{G}^{\circ}\right)$ and $\operatorname{Irr}_{\mathbb{C}}\left(W_{\mathfrak{t}^{\circ}}\right)$ relates to part (a) by

$$
\left.{ }^{L} \Sigma_{\mathfrak{t}_{\phi}}(\phi, \rho)\right|_{W_{\mathfrak{t}_{\phi}^{\circ}}}=\bigoplus_{i} \Sigma_{\mathfrak{t}_{\phi}^{\circ}}\left(u_{\phi}, \rho_{i}\right)
$$

where $\rho=\bigoplus_{i} \rho_{i}$ is a decomposition into irreducible $A_{\mathcal{G}_{\phi}^{\circ}}\left(u_{\phi}\right)$-subrepresentations.

(c) The $\mathbf{G}^{\vee}$-conjugacy class of $\left(\left.\phi\right|_{W_{F}}, u_{\phi}, \rho_{i}\right)$ is determined by any irreducible $\mathbb{C}\left[W_{\mathfrak{t}_{\phi}^{\circ}}\right]$ subrepresentation of ${ }^{L} \sum_{\mathfrak{t}_{\phi}}(\phi, \rho)$.

\section{A Galois version of the ABPS Conjecture}

7.1. Twisted extended quotients. Let $\Gamma$ be a group acting on a topological space $X$, and let $\downarrow$ be a given function which assigns to each $x \in X$ a 2-cocycle

$$
\natural_{x}: \Gamma_{x} \times \Gamma_{x} \rightarrow \mathbb{C}^{\times} \text {, where } \Gamma_{x}=\{\gamma \in \Gamma: \gamma x=x\} .
$$

We assume that $\natural_{\gamma x}$ and $\gamma_{*} \natural_{x}$ define the same class in $H^{2}\left(\Gamma_{\gamma x}, \mathbb{C}^{\times}\right)$, where $\gamma_{*}: \Gamma_{x} \rightarrow$ $\Gamma_{\gamma x}$ sends $\alpha$ to $\gamma \alpha \gamma^{-1}$. Let $\mathbb{C}\left[\Gamma_{x}, \natural_{x}\right]$ be the corresponding twisted algebra as defined in Section 4.3. We set

$$
\widetilde{X}_{\natural}:=\left\{(x, E): x \in X, E \in \operatorname{Irr} \mathbb{C}\left[\Gamma_{x}, \natural_{x}\right]\right\} .
$$


and we topologize it by decreeing that a subset of $\widetilde{X}_{\natural}$ is open if and only if its projection to the first coordinate is open in $X$.

We require, for every $(\gamma, x) \in \Gamma \times X$, a definite algebra isomorphism

$$
\phi_{\gamma, x}: \mathbb{C}\left[\Gamma_{x}, \natural_{x}\right] \rightarrow \mathbb{C}\left[\Gamma_{\gamma x}, \natural_{\gamma x}\right]
$$

such that:

- if $\gamma x=x$, then $\phi_{\gamma, x}$ is conjugation by an element of $\mathbb{C}\left[\Gamma_{x}, \natural_{x}\right]^{\times}$;

- $\phi_{\gamma^{\prime}, \gamma x} \circ \phi_{\gamma, x}=\phi_{\gamma^{\prime} \gamma, x}$ for all $\gamma^{\prime}, \gamma \in \Gamma, x \in X$.

Then we can define a $\Gamma$-action on $\tilde{X}_{\natural}$ by

$$
\gamma \cdot(x, E):=\left(\gamma x, E \circ \phi_{\gamma, x}^{-1}\right) .
$$

We form the twisted extended quotient

$$
(X / / \Gamma)_{\natural}:=\tilde{X}_{\natural} / \Gamma .
$$

Remark 7.1. Furthermore we note that $(X / / \Gamma)_{\natural}$ reduces to the extended quotient of the second kind $(X / / \Gamma)_{2}$ from [ABPS6, $\left.\S 2\right]$ if $\natural_{x}$ is trivial for all $x \in X$ and $\phi_{\gamma, x}$ is conjugation by $\gamma$.

The extended quotient of the second kind is an extension of the ordinary quotient in the sense that it keeps track of the duals of the isotropy groups. Namely, in $(X / / \Gamma)_{2}$ every point $x \in X / \Gamma$ has been replaced by the set $\operatorname{Irr}\left(\Gamma_{x}\right)$.

\subsection{The Bernstein decomposition of the category of smooth representa-} tions.

Let $\operatorname{Rep}(G)$ denote the category of smooth representations of $G$. Let $P$ be a parabolic subgroup of $G$ and let $L$ be a Levi factor of $P$. Let $\sigma$ be a supercuspidal irreducible representation of $L$. We call $(L, \sigma)$ a cuspidal pair, and we consider such pairs up to inertial equivalence: this is the equivalence relation generated by:

- unramified twists, $(L, \sigma) \sim(L, \sigma \otimes \chi)$ for $\chi \in X_{\mathrm{nr}}(L)$, where $X_{\mathrm{nr}}(L)$ is the group of unramified (not necessarily unitary) characters $L \rightarrow \mathbb{C}^{\times}$;

- $G$-conjugation, $(L, \sigma) \sim\left(g L g^{-1}, g \cdot \sigma\right)$ for $g \in G$.

We denote a typical inertial equivalence class by $\mathfrak{s}=[L, \sigma]_{G}$. In particular

$$
\mathfrak{s}_{L}:=[L, \sigma]_{L}=\left\{\sigma \otimes \chi \in \operatorname{Irr}(L): \chi \in X_{\mathrm{nr}}(L)\right\} .
$$

Bernstein attached to every $\mathfrak{s}$ a block in the category $\operatorname{Rep}(G)$, in the following way. Denote the normalized parabolic induction functor by $\mathrm{I}_{P}^{G}$. We define

$$
\begin{aligned}
& \operatorname{Irr}(G)^{\mathfrak{s}}=\left\{\pi \in \operatorname{Irr}(G): \pi \text { is a constituent of } \mathrm{I}_{P}^{G}(\sigma \otimes \chi) \text { for some } \sigma \in \mathfrak{s}_{L}\right\} \\
& \operatorname{Rep}(G)^{\mathfrak{s}}=\left\{\pi \in \operatorname{Rep}(G): \text { every irreducible constituent of } \pi \text { belongs to } \operatorname{Irr}(G)^{\mathfrak{s}}\right\} .
\end{aligned}
$$

We denote the set of all inertial equivalence classes for $G$ by $\mathfrak{B}(G)$.

Theorem 7.2. [BeDe, Proposition 2.10]

The category $\operatorname{Rep}(G)$ decomposes as

$$
\operatorname{Rep}(G)=\prod_{\mathfrak{s} \in \mathfrak{B}(G)} \operatorname{Rep}(G)^{\mathfrak{s}} .
$$

The space of irreducible G-representations is a disjoint union

$$
\operatorname{Irr}(G)=\bigsqcup_{\mathfrak{s} \in \mathfrak{B}(G)} \operatorname{Irr}(G)^{\mathfrak{s}} .
$$

Notation 7.3. Let $\operatorname{Irr}_{\text {cusp }}(L)$ be the set of isomorphism classes of supercuspidal irreducible smooth representations of $L$. 
For $\sigma \in \operatorname{Irr}_{\text {cusp }}(L)$ (and in fact for every irreducible $L$-representation) the group

$$
X_{\mathrm{nr}}(L, \sigma):=\left\{\chi \in X_{\mathrm{nr}}(L): \sigma \otimes \chi \cong \sigma\right\}
$$

is finite. Thus there is a bijection

$$
X_{\mathrm{nr}}(L) / X_{\mathrm{nr}}(L, \sigma) \rightarrow \operatorname{Irr}(L)^{\mathfrak{s}_{L}}: \chi \mapsto \sigma \otimes \chi,
$$

which endows $\operatorname{Irr}(L)^{\mathfrak{s}_{L}}$ with the structure of a complex torus. Up to isomorphism this torus depends only on $\mathfrak{s}$, and it is known as the Bernstein torus $T_{\mathfrak{s}}$ attached to $\mathfrak{s}$. We note that $T_{\mathfrak{s}}$ is only an algebraic variety, it is not endowed with a natural multiplication map. In fact it does not even possess an unambigous "unit", because in general there is no preferred choice of an element $\sigma \in \mathfrak{s}_{L}$.

The group $W(G, L):=\mathrm{N}_{G}(L) / L$ acts on $\operatorname{Irr}(L)$ by

$$
w \cdot \pi=\left[\bar{w} \cdot \pi: l \mapsto \pi\left(\bar{w}^{-} l \bar{w}\right)\right] \quad \text { for any lift } \bar{w} \in \mathrm{N}_{G}(L) \text { of } w \in W(G, L) .
$$

Bernstein also associated to $\mathfrak{s}$ the finite group

$$
W_{\mathfrak{s}}:=\left\{w \in W(G, L): w \cdot \operatorname{Irr}(L)^{\mathfrak{s}_{L}}=\operatorname{Irr}(L)^{\mathfrak{s} L}\right\} .
$$

It acts naturally on $T_{\mathfrak{s}}$, by automorphisms of algebraic varieties.

Closely related to the Bernstein decomposition is the theory of the Bernstein center. By [BeDe, Théorème 2.13] the categorical centre of the Bernstein block $\operatorname{Rep}^{\mathfrak{s}}(G)$ is

$$
\mathrm{Z}\left(\operatorname{Rep}(G)^{\mathfrak{s}}\right) \cong \mathcal{O}\left(T_{\mathfrak{s}}\right)^{W_{\mathfrak{s}}}=\mathcal{O}\left(T_{\mathfrak{s}} / W_{\mathfrak{s}}\right) .
$$

Here $\mathcal{O}$ stands for the regular functions on an affine variety. Moreover the map

$$
\text { sc: } \operatorname{Irr}(G)^{\mathfrak{s}} \rightarrow T_{\mathfrak{s}} / W_{\mathfrak{s}}
$$

induced by (51) is surjective and has finite fibers [BeDe, §3]. Theorem 7.2 implies that every $\pi \in \operatorname{Irr}(G)$ is a constituent of $I_{P}^{G}(\sigma)$, where $[L, \sigma]_{G}$ is uniquely determined. By (51) the supercuspidal $L$-representation $\sigma \in T_{\mathfrak{s}}$ is in fact uniquely determined up to $W_{\mathfrak{s}}$. The map $\pi \mapsto W_{\mathfrak{s}} \sigma$ is just $\mathbf{s c}$, and for this reason it is called the cuspidal support map. Via this map $\operatorname{Irr}^{\mathfrak{s}}(G)$ can be regarded as a non-separated algebraic variety lying over $T_{\mathfrak{s}} / W_{\mathfrak{s}}$.

7.3. The ABPS Conjecture. Let $\mathfrak{s}=[L, \sigma]_{G}$ be an inertial equivalence class for $G$. Let $W_{\mathfrak{s}, t}$ be the stabilizer in $W_{\mathfrak{s}}$ of a point $t \in T_{\mathfrak{s}}$.

The ABPS conjecture from [ABPS2, §15] and [ABPS7, Conjecture 2] in its roughest form asserts that there exists a family of 2-cocycles

$$
\natural_{t}: W_{\mathfrak{s}, t} \times W_{\mathfrak{s}, t} \rightarrow \mathbb{C}^{\times} \quad t \in T_{\mathfrak{s}},
$$

and a bijection

$$
\operatorname{Irr}(G)^{\mathfrak{s}} \longleftrightarrow\left(T_{\mathfrak{s}} / / W_{\mathfrak{s}}\right)_{\mathfrak{\natural}}
$$

such that:

- it restricts to a bijection between tempered representations and the unitary part of the extended quotient (as explained below);

- it is canonical up to permutations within $L$-packets, that is, for any $\phi \in$ $\Phi(G)$, the image of $\Pi_{\phi}(G) \cap \operatorname{Irr}^{\mathfrak{s}}(G)$ is canonically defined (assuming a LLC for $G$ exists). 
The set $\operatorname{Irr}_{\text {cusp }}(L)$ of supercuspidal $L$-representations is stable under the $W(G, L)$ action (49). The definitions of $W_{\mathfrak{s}}$ and of extended quotients imply that for a fixed Levi subgroup $L$ of $G$ there is a natural bijection

$$
\bigsqcup_{\mathfrak{s}=[L, \sigma]_{G}}\left(T_{\mathfrak{s}} / / W_{\mathfrak{s}}\right)_{\mathfrak{\natural}} \rightarrow\left(\operatorname{Irr}_{\text {cusp }}(L) / / W(G, L)\right)_{\mathfrak{\natural}} .
$$

In view of Theorem 7.2 , the ABPS-Conjecture can also be formulated in terms of a bijection

$$
\operatorname{Irr}(G) \longleftrightarrow \bigsqcup_{L}\left(\operatorname{Irr}_{\text {cusp }}(L) / / W(G, L)\right)_{\natural},
$$

where $L$ runs through a set of representatives for the $G$-conjugacy classes of Levi subgroups of $G$. In this version, our conjecture asserts that $\operatorname{Irr}(G)$ is determined by a much smaller set of data, namely the supercuspidal representations of Levi subgroups $L$ of $G$, and the actions of the Weyl groups $W(G, L)$ on those.

It is expected that the group cohomology classes $\natural_{t} \in H^{2}\left(W_{\mathfrak{s}, t}, \mathbb{C}^{\times}\right)$reflect the character of $\mathrm{Z}\left(G_{\mathrm{sc}}^{\vee}\right)^{W_{F}}$ which via the Kottwitz isomorphism determines how $G$ is an inner twist of a quasi-split group. In particular $\square$ should be trivial whenever $G$ is quasi-split. The simplest known example of a nontrivial cocycle involves a non-split inner form of $\mathrm{SL}_{10}(F)$ [ABPS5, Example 5.5]. That example also shows that it is sometimes necessary to use twisted extended quotients in the ABPS Conjecture.

7.4. A version for enhanced $L$-parameters. The main goal of this section is to state an analogue of (53) and (55) for enhanced Langlands parameters.

We will recall first the statement of [AMS1, Lemma. 7.4]. There exists a character $\zeta_{G} \in \operatorname{Irr}\left(\mathrm{Z}\left(\mathbf{G}_{\mathrm{sc}}^{\vee}\right)\right)$ such that: $\left.\zeta_{G}\right|_{\mathrm{Z}\left(\mathbf{G}_{\mathrm{sc}}^{\vee}\right)^{W_{F}}}$ parametrizes the inner twist $G$ via the Kottwitz isomorphism (9), and $\zeta_{G}=1$ on $\mathrm{Z}\left(\mathbf{G}_{\mathrm{sc}}^{\vee}\right) \cap \mathrm{Z}\left(\mathbf{L}_{\mathrm{c}}^{\vee}\right)^{\circ}$, for every Levi subgroup $L$ of $G$. We set

$$
\Phi_{\mathrm{e}, \zeta_{G}}:=\Phi_{\mathrm{e}}(G),
$$

where $\Phi_{\mathrm{e}}(G)$ is as in 3.6 . Let $L \subset G$ be a Levi subgroup and let $\phi: W_{F} \times \mathrm{SL}_{2}(\mathbb{C}) \rightarrow$ ${ }^{L} L$ be a Langlands parameter for $L$. There exists a natural injection $\mathcal{R}_{\phi}^{\mathcal{L}} \rightarrow \mathcal{R}_{\phi}$. We extend the character $\zeta_{G}$ to a character of $\mathrm{Z}\left(\mathbf{G}_{\mathrm{sc}}^{\vee}\right) \mathrm{Z}\left(L_{\mathrm{c}}^{\vee}\right)^{\circ}$ which is trivial on $\mathrm{Z}\left(L_{\mathrm{c}}^{\vee}\right)^{\circ}$, and denote by $\zeta_{G}^{L}$ the restriction of the latter character to $\mathrm{Z}\left(\mathbf{L}_{\mathrm{sc}}^{\vee}\right)$.

The following result is proved in [Mou2, Theorem 3.3] for $G$ a split classical group, and in AMS1, Theorem 9.3] for general $G$.

Theorem 7.4. AMS1, Theorem 9.3]

(a) Let $\mathfrak{s}_{L}^{\vee}=\left[{ }^{L} L, \phi_{v}, q \epsilon\right]_{L_{L}}$ be an $\mathcal{H}$-relevant inertial equivalence class for the Levi $L$ subgroup ${ }^{L} L$ of ${ }^{L} \mathcal{H}$ and recall the notations (47). The maps ${ }^{L} \Sigma_{\mathfrak{t}}$ from Proposition 6.10. a combine to a bijection

$$
\begin{array}{ccc}
\Phi_{\mathrm{e}}\left({ }^{L} G\right)^{\mathfrak{s}^{\vee}} & \longleftrightarrow & \left(\Phi_{\mathrm{e}}\left({ }^{L} L\right)^{\mathfrak{s}^{\vee}} / / W_{\mathfrak{s}^{\vee}}\right)_{\kappa} \\
(\phi, \rho) & \mapsto & \left({ }^{L} \Psi(\phi, \rho), \Sigma_{\mathfrak{t}}\left(u_{\phi}, \rho\right)\right) \\
\left(\left.\phi_{v}\right|_{W_{F}}, \Sigma_{\mathfrak{t}}^{-1}(\tau)\right) & \longleftrightarrow & \left({ }^{L} L, \phi_{v}, \varepsilon, \tau\right) .
\end{array}
$$

(b) The bijection from part (a) has the following properties:

- It preserves boundedness of (enhanced) L-parameters.

- The restriction of $\tau$ to $W_{\mathfrak{t}^{\circ}}$ canonically determines the (non-enhanced) $L$ parameter in ${ }^{L} \Sigma_{\mathfrak{t}}(\tau)$. 
- Let $z, z^{\prime} \in X_{\mathrm{nr}}\left({ }^{L} L\right)$ and let $\Gamma \subset W_{\mathfrak{s}^{\vee}, z \phi_{v}, \epsilon}$ be a subgroup. Suppose that $\Gamma=\bar{\Gamma} / L \cong \bar{\Gamma}_{\mathrm{c}} / L_{\mathrm{c}}$, where

$\bar{\Gamma} \subset \mathrm{N}_{\mathbf{G}^{\vee}}\left({ }^{L} L\right) \cap \mathrm{Z}_{\mathbf{G}^{\vee}}^{1}\left(\left.z^{\prime} \phi\right|_{W_{F}}\right) \quad$ with preimage $\quad \bar{\Gamma}_{c} \subset \mathrm{Z}_{\mathbf{G}_{\mathrm{sc}}^{\vee}}\left(z^{\prime} \phi\left(W_{F}\right)\right)^{\circ}$.

Then the 2-cocycle $\kappa_{\mathfrak{s} \vee}, z \phi_{v}, \varepsilon$ is trivial on $\Gamma$.

(c) Let $\zeta_{G} \in \operatorname{Irr}\left(\mathrm{Z}\left(\mathbf{G}_{\mathrm{sc}}^{\vee}\right)\right)$. We write

$$
\Phi_{\mathrm{e}, \zeta_{G}}(G, L):=\left\{(\phi, \rho) \in \Phi_{e, \zeta_{G}}(G): \mathbf{S c}(\phi, \rho) \in \Phi_{\text {cusp }}(L)\right\} .
$$

The bijections from part (a) give a bijection

$$
\Phi_{\mathrm{e}, \zeta_{G}}(G, L) \longleftrightarrow\left(\Phi_{\text {cusp }, \zeta_{G}^{L}}(L) / / W(G, L)\right)_{\kappa} .
$$

(d) Let $\mathfrak{L} \mathfrak{e v}(G)$ be a set of representatives for the conjugacy classes of Levi subgroups of $G$. The maps from part (c) combine to a bijection

$$
\Phi_{\mathrm{e}, \zeta_{G}}(G) \longleftrightarrow \bigsqcup_{L \in \mathfrak{L} \mathfrak{v}(G)}\left(\Phi_{\operatorname{cusp}, \zeta_{G}^{L}}(L) / / W(G, L)\right)_{\kappa} .
$$

(e) Assume that $\mathrm{Z}\left(\mathbf{L}_{\mathrm{sc}}^{\vee}\right)$ is fixed by $W_{F}$ for every Levi subgroup $L \subset G$ (for instance, $G$ is an inner twist of a split group). Recall that $G_{\vartheta}$ is the inner twist of $G$ determined by $\vartheta \in H^{1}\left(F, G_{\mathrm{ad}}\right) \cong \operatorname{Irr}_{\mathbb{C}}\left(\mathrm{Z}\left(\mathbf{G}_{\mathrm{sc}}^{\vee}\right)^{W_{F}}\right)$. The union of part (d) for all such $\vartheta$ is a bijection

$$
\Phi_{\mathrm{e}}\left({ }^{L} G\right) \longleftrightarrow \bigsqcup_{\vartheta \in H^{1}\left(F, G_{\mathrm{ad}}\right)} \bigsqcup_{\vartheta} \in \bigsqcup_{\mathfrak{L v}\left(G_{\vartheta}\right)}\left(\Phi_{\text {cusp }}\left(L_{\vartheta}\right) / / W\left(G_{\vartheta}, L_{\vartheta}\right)\right)_{\kappa} .
$$

7.5. A conjectural diagram. The above result leads to the following conjecture stated in AMS1:

Conjecture 7.5. There exists a commutative bijective diagram

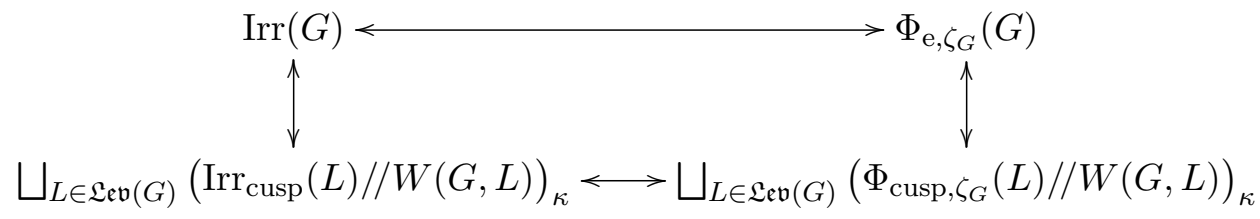

with the following maps:

- the right hand side is Theorem 7.4.

- the upper horizontal map is a local Langlands correspondence for $G$,

- the lower horizontal map is obtained from local Langlands correspondences for $\operatorname{Irr}_{\text {cusp }}(L)$ by applying $(\cdot / / W(G, L))_{\kappa}$,

- the left hand side is the bijection in the ABPS conjecture [ABPS7, § 2].

With this conjecture one can reduce the problem of finding a LLC for $G$ to that of finding local Langlands correspondences for supercuspidal representations of its Levi subgroups. Conjecture 7.5 is currently known in the following cases:

- inner forms of $\mathrm{GL}_{n}(F)$ [ABPS4, Theorem 5.3],

- inner forms of $\mathrm{SL}_{n}(F)$ [ABPS4, Theorem 5.6],

- split classical groups [Mou1, §5.3],

- principal series representations of split groups [ABPS6, § 16]. 


\section{REFERENCES}

[AHJR] P. Achar, A. Henderson, D. Juteau, S. Riche, "Modular generalized Springer correspondence I: the general linear group", J. Eur. Math. Soc. (JEMS) 18 (2016), no. 7, 1405-1436.

[Ar1] J. Arthur, "A note on L-packets", Pure Appl. Math. Quaterly 2.1 (2006), 199-217.

[Ar2] _ The endoscopic classification of representations: orthogonal and symplectic groups, Colloquium Publications volume 61, American Mathematical Society, 2013.

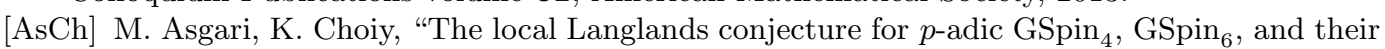
inner forms", Forum Math. 29 (2017), no. 6, 1261-1290.

[Au] A.-M. Aubert, "Around the Langlands program", Jahresber. Dtsch. Math.-Ver. 120 (2018), no. $1,3-40$.

[ABPS1] A.-M. Aubert, P.F. Baum, R.J. Plymen, M. Solleveld, "On the local Langlands correspondence for non-tempered representations", Münster J. Math. 7 (2014), no. 1, 27-50.

[ABPS2] _ _ "Geometric structure in smooth dual and local Langlands correspondence", Japan. J. Math. 9 (2014), 99-136.

[ABPS3] _ "Depth and the local Langlands correspondence", in Arbeitstagung Bonn 2013, In Memory of Friedrich Hirzebruch, eds. W. Ballmann, C. Blohmann, G. Faltings, P. Teichner, et D. Zagier pp. 17-41, Progr. Math. 319, Birkhäuser/Springer, 2016.

[ABPS4] _ , "The local Langlands correspondence for inner forms of $\mathrm{SL}_{n}$ ", Res. Math. Sci. 3 (2016), Paper No. 32, 34 pp.

[ABPS5] _ _Hecke algebras for inner forms of $p$-adic special linear groups", J. Inst. Math. Jussieu 16 (2017), no. 2, 351-419.

[ABPS6] _ "The principal series of $p$-adic groups with disconnected centre", Proc. Lond. Math. Soc. (3) 114 (2017), no. 5, 798-854.

[ABPS7] _ "Conjectures about $p$-adic groups and their noncommutative geometry", in Around Langlands correspondences, 15-51, Contemp. Math. 691, Amer. Math. Soc., Providence, RI, 2017.

[AMPS] A.-M. Aubert, S. Mendes, R. Plymen, M. Solleveld, "L-packets and depth for $\mathrm{SL}_{2}(K)$ with $K$ a local function field of characteristic 2", Int. J. Number Theory 13 (2017), no. 10, $2545-2568$.

[AMS1] A.-M. Aubert, A. Moussaoui, M. Solleveld, "Generalizations of the Springer correspondence and cuspidal Langlands parameters", Manuscripta Math. 1-72, DOI 10.1007/s00229-018-1001$8,2018$.

[AMS2] _ _ "Graded Hecke algebras for disconnected reductive groups", to appear in the Proceedings of the Simons Symposium 2017 Geometric Aspects of the Trace Formula.

[AMS3] _ "Affine Hecke algebras for Langlands parameters", arXiv:1701.03593.

[BeDe] J. Bernstein, P. Deligne, "Le "centre" de Bernstein", pp. 1-32 in: Représentations des groupes réductifs sur un corps local, Travaux en cours, Hermann, 1984.

[BeLu] J. Bernstein, V. Lunts, "Equivariant sheaves and functors", Lecture Notes in Mathematics 1578, Springer-Verlag, 1994.

[Bor] A. Borel, "Automorphic L-functions", Proc. Symp. Pure Math 33.2 (1979), 27-61.

[BuHe] C. Bushnell, G. Henniart, "The essentially tame local Langlands correspondence, III: the general case", Proc. Lond. Math. Soc. (3) 101 (2010), no. 2, 497-553.

[Ch] K. Choiy, "The local Langlands conjecture for the $p$-adic inner form of $\mathrm{Sp}_{4}$ ", Int. Math. Res. Not. IMRN 2017 6, 1830-1889.

[CuRe] C.W. Curtis, I. Reiner, Representation theory of finite groups and associative algebras, Pure and Applied Mathematics 11, John Wiley \& Sons, 1962.

[DeRe] S. DeBacker, M. Reeder, "Depth-zero supercuspidal L-packets and their stability", Ann. of Math. (2) 169.3 (2009), 795-901.

[FOS] Y. Feng, E. Opdam, M. Solleveld, "Supercuspidal unipotent representations: $L$-packets and formal degrees", arXiv:1805.01888.

[GaTak1] W.T. Gan, S. Takeda, "The local Langlands conjecture for GSp(4)", Ann. of Math. (2) 173 (2011), no. 3, 1841-1882.

[GaTak2] _ "The local Langlands conjecture for Sp(4)", Int. Math. Res. Not. IMRN (2010) 15, 2987-3038.

[GaTan] W.T. Gan, W. Tantono, "The local Langlands conjecture for GSp(4), II: The case of inner forms", Amer. J. Math. 136 (2014), no. 3, 761-805. 
[Ga] R. Ganapathy, "The local Langlands correspondence for GSp 4 over local function fields", Amer. J. Math. 137 (2015), no. 6, 1441-1534.

[GaVa] R. Ganapathy, S. Varma, "On the local Langlands correspondence for split classical groups over local function fields", J. Inst. Math. Jussieu 16 (2017), no. 5, 987-1074.

[GoJa] R. Godement, H. Jacquet, Zeta functions of simple algebras, Lecture Notes in Mathematics 260, Springer-Verlag, 1972.

[GrRe] B.H. Gross, M. Reeder, Arithmetic invariants of discrete Langlands parameters, Duke Math. J. 154 (2010), 431-508.

[Hai] T.J. Haines, "The stable Bernstein center and test functions for Shimura varieties", pp. 118186 in: Automorphic Forms and Galois Representations, London Mathematical Society Lecture Note Series 415, Cambrige University Press, 2014.

[HaTa] M. Harris, R. Taylor, The geometry and cohomology of some simple Shimura varieties, Annals of Math. Studies 151, Princeton University Press, 2001.

[Hen1] G. Henniart, "Caractérisation de la correspondance de Langlands locale par les facteurs $\epsilon$ de paires, Invent. Math. 113 (1993), 339-350.

[Hen2] _ "Une preuve simple des conjectures de Langlands pour GL $(n)$ sur un corps $p$ adique", Inventiones Mathematicae 139, 439-455, 2000.

[HiSa] K. Hiraga, H. Saito, "On $L$-packets for inner forms of $\mathrm{SL}_{n}$ ", Mem. Amer. Math. Soc. 1013, Vol. 215 (2012).

[JN] D. Jiang, C. Nien, "On the local Langlands conjecture and related problems over p-adic local fields", Proceedings of the Sixth International Congress of Chinese Mathematicians. Vol. I, 309-325, Adv. Lect. Math. (ALM) 36, Int. Press, Somerville, MA, 2017.

[Kal1] T. Kaletha, "Global rigid inner forms and multiplicities of discrete automorphic representations", arXiv:1501.01667, 2015.

[Kal2] _ _ "The local Langlands conjectures for non-quasi-split groups", in Families of automorphic forms and the trace formula, 217-257, Simons Symp., Springer, 2016.

[KMSW] T. Kaletha, A. Minguez, S. W. Shin, P.-J. White, "Endoscopic Classification of Representations: Inner Forms of Unitary Groups", arXiv:1409.3731.

[Ko] R. Kottwitz, "Stable trace formula: cuspidal tempered terms", Duke Math. J. 51 (1984), no. $3,611-650$.

[Lan] R.P. Langlands, "Representations of abelian algebraic groups", pp. 231-250 in: Olga TausskyTodd: in memoriam, Pacific J. Math., Special Issue, 1997.

[LRS] G. Laumon, M. Rapoport, U. Stuhler, "D-elliptic sheaves and the Langlands correspondence", Invent. Math. 113 (1993), 217-238.

[Lom] L. Lomeli, "Langlands program and Ramanujan conjecture: a survey", Preprint 2018.

[Lus1] G. Lusztig, "Some examples of square-integrable representations of semisimple $p$-adic groups", Trans. Amer. Math. Soc. 277 (1983), 623-653.

[Lus2] _ "Intersection cohomology complexes on a reductive group", Invent. Math. 75.2 (1984), 205-272.

[Lus3] _ "Fourier transforms on a semisimple Lie algebra over $\mathbb{F}_{q}$ ", in Algebraic Groups (Utrecht 1986), Lecture Notes in Math. 1271, Springer, Berlin, 177-188 (1987).

[Lus4] _ "Classification of unipotent representations of simple p-adic groups", Int. Math. Res. Notices 11 (1995), 517-589.

[Lus5] _ "Classification of unipotent representations of simple $p$-adic groups. II", Represent. Theory 6 (2002), 243-289.

[Lus6] _ "Character sheaves on disconnected groups. V", Represent. Theory 8, 346-376 (2004).

[MiPa] M. Mishra, B. Patanayak, "A note on depth preservation”, Preprin 2018.

[Moe] C. Mœglin, "Stabilité en niveau zéro, pour les groupes orthogonaux impairs p-adiques", Documenta Math. 9 (2004), 527-564.

[MW1] C. Møglin, J.-L. Waldspurger, Stabilisation de la formule des traces tordue, Vol. 316-317, Progress in Math., Birkhäuser Boston, 2017.

[Mok] C.P. Mok, Endoscopic classification of representations of quasi-split unitary groups, Mem. Amer. Math. Soc. 235 (2015), no. 1108, vi+248 pp.

[Mou1] A. Moussaoui, "Centre de Bernstein dual pour les groupes classiques", Represent. Theory 21 (2017), 172-246. 
[Mou2] _ "Proof of the Aubert-Baum-Plymen-Solleveld conjecture for split classical groups", in Around Langlands correspondences, 257-281, Contemp. Math., 691, Amer. Math. Soc., Providence, RI, 2017.

[MoPr] A. Moy, G. Prasad, "Jacquet functors and unrefined minimal K-types", Comment. Math. Helv. 71 (1996), no. 1, 98-121.

[Ng1] B.C. Ngô, "Fibration de Hitchin et endoscopie", Invent. Math. (2006), 399-453.

[Ng2] _ _Le lemme fondamental pour les algèbres de Lie", Publ. Math. Inst. Hautes Études Sci. 111 (2010), 1-169.

[Oi] M. Oi, "Depth preserving property of the local Langlands correspondence for quasi-split classical groups in a large residual characteristic", arXiv:1804.10901.

[Scho] P. Scholze, "The local Langlands correspondence for $\mathrm{GL}_{n}$ over $p$-adic fields", Invent. Math. 192 (2013), 663-715.

[Ser] J.-P. Serre, Corps locaux. Hermann, Paris, 1962.

[SZ] A. Silberger, W. Zink, "Langlands classification for $L$-parameters", to appear in Journal of Algebra.

[Spr] T.A. Springer, Linear algebraic groups 2nd ed., Progress in Mathematics 9, Birkhäuser, 1998.

[Tat] J. Tate, "Number theoretic background", Proc. Symp. Pure Math 33.2 (1979), 3-26.

[Vog] D. Vogan "The local Langlands conjecture", pp. 305-379 in: Representation theory of groups and algebras, Contemp. Math. 145, American Mathematical Society, 1993.

[Wa] J.-L. Waldspurger, "Le lemme fondamental implique le transfert, Compositio Math. 105 (1997), no. 2, 153-236.

[Xu] B. Xu, "On a lifting problem of L-packets", Compos. Math. 152 (2016), no. 9, 1800-1850.

[Yu] J.-K. Yu, "On the local Langlands correspondence for tori", pp. 177-183 in: Ottawa lectures on admissible representations of reductice p-adic groups, Fields Institute Monographs, American Mathemical Society, 2009

[Zel] A.V. Zelevinsky, "Induced representations of reductive $p$-adic groups II. On irreducible representations of GL(n)", Ann. Sci. École Norm. Sup. (4) 13.2 (1980), 165-210.

C.N.R.S., Sorbonne Université, Université Paris Diderot, Institut de Mathématiques De Jussieu - Paris Rive Gauche, IMJ-PRG, F-75005 Paris, France

Email address: anne-marie.aubert@imj-prg.fr 